\title{
Screening Two Lycopersicon peruvianum Collections for Resistance to Tomato spotted wilt virus
}

Luis F. Gordillo and Mikel R. Stevens, Department of Plant and Wildlife Sciences, Brigham Young University, Provo, UT 84602; Mark A. Millard, Department of Geology, Brigham Young University-Idaho, Rexburg, ID 83460; and Brad Geary, Department of Plant and Wildlife Sciences, Brigham Young University, Provo

\begin{abstract}
Gordillo, L. F., Stevens, M. R., Millard, M. A., and Geary, B. 2008. Screening two Lycopersicon peruvianum collections for resistance to Tomato spotted wilt virus. Plant Dis. 92:694-704.

The United States Department of Agriculture (USDA) Research Service and the Tomato Genetics Resource Center (TGRC) Lycopersicon peruvianum germplasm collections (16,335 plants from 285 accessions) were screened with the Tomato spotted wilt virus (TSWV) isolates TSWV6 from Hawaii, and $\mathrm{An}_{\mathrm{wa}}-1$ from Western Australia. Using TSWV6 to screen for resistance, 10,634 L. peruvianum plants from 280 accessions were screened for resistance, resulting in $168(60 \%)$ accessions with 1,437 (14\%) plants indicating resistance, with all 1,404 89S ( $\mathrm{Sw}$ $\left.5^{+} / S w-5^{+}\right)$and $1,45689 \mathrm{R}(S w-5 / S w-5)$ controls infected. When using $\mathrm{An}_{\mathrm{wa}}-1$ for screening, 864 $(15 \%)$ of 5,701 L. peruvianum plants were uninfected from 106 of the 181 accessions tested, and $472(95 \%)$ of the $49589 \mathrm{~S}$ and $421(73 \%)$ of the $57489 \mathrm{R}$ controls were infected. Of the 172 accessions tested with both isolates, 54 were resistant to one isolate but not the other. Additionally, more accessions from the USDA than from the TGRC collection indicated resistance. TSWV-resistant accessions were somewhat equally distributed throughout the $L$. peruvianum geographic range, with an observation that northern Chile and southern Peru seemed to have an unusually high portion of accession indicating resistance. The value of $S w-5$ is discussed in relationship to potential additional sources of TSWV resistance.
\end{abstract}

Additional keywords: Lycopersicon esculentum, thrips, Tospovirus

Tomato spotted wilt virus (TSWV), classified as a Tospovirus, infects cultivated tomato (Lycopersicon esculentum Mill.), causing plant stunting, necrotic leaf spotting, mottling, epinasty, plant and fruit deformity, and, in severe cases, death $(3,7)$. Thrips are the natural vectors of tospoviruses, with species of the genus Frankliniella and Thrips being the most common $(8,9,32,43)$. Because of the globalization of trade these insects have spread worldwide along with their vectored disease $(4,28,44,57,60-62)$. Tospoviruses infect at least 15 monocotyledonous and 69 dicotyledonous families, of which Asteraceae, Solanaceae, and Fabaceae are most com-

Corresponding author: M. R. Stevens

E-mail: mikel_stevens@byu.edu

Current address of L. F. Gordillo: Biology Department, Snow College, 150 East, College Avenue, Snow College, Ephraim, UT 84627.

Current address of M. A. Millard: Department of Geology, One Bear Place \#97354, Baylor University, Waco, TX 76798.

Accepted for publication 3 January 2008.

doi:10.1094/PDIS-92-5-0694

This article is in the public domain and not copyrightable. It may be freely reprinted with customary crediting of the source. The American Phytopathological Society, 2008. monly affected. More than 1,090 plant species have been reported to be infected by this virus, including many crops of economic importance, with vegetable yield losses worldwide estimated at one billion dollars annually (32). In the 2000 growing season, TSWV epidemics in tomato were estimated to cause $\$ 8.8$ million in losses in Georgia (43). Cho et al. (7) reported that yield losses in Hawaii have been high enough to cease tomato production in some areas due to TSWV. In Brazil, Argentina, Spain, Portugal, and Italy, it is becoming the most limiting factor for tomato production $(1,2,4,30,38,51,65)$.

Growers have tried to manage TSWV by controlling thrips through different approaches such as crop rotation, leaving the land fallowed, eliminating crop residue, insecticides, weed control, and reflective plastic mulch $(29,42)$. However thrips are minute winged insects that easily migrate into fields, regardless of grower deterrents, then infect plants before insecticides have time to control them effectively (8). Of all the cultural practices tried, weed control and reflective mulches have had the most positive effect on increased marketable tomato yields $(29,42,43)$.

A viable method to control TSWV is through natural resistance found in wild relatives of domesticated tomato plants, which provide a possible control to TSWV and subsequent reduction in fruit damage
$(8,43)$. Stevens et al. (58) screened 188 accessions of seven different Lycopersicon spp. for resistance to isolates of TSWV, finding that L. peruvianum (L.) Mill. and L. chilense Dun. provided the highest level of resistance to TSWV. All available $L$. chilense accessions and 12 L. peruvianum accessions were screened for resistance from the United States germplasm collections $(54,58)$. Additionally, a number of other reports $(4,10-12,15,18,20,23,24,27$, $31,33,47,49,50,52,53,62,63)$ have identified a high level of TSWV resistance in $L$. peruvianum beginning as early as 1939 (64). In 1946, Norris reported in relationship to TSWV, that " $L$. peruvianum possesses true resistance amounting almost to immunity" (31). Despite this observation, no comprehensive study of the L. peruvianum U.S. germplasm collections has been conducted, even though the TSWV resistance gene " $S w-5$ " was introgressed into the cultivated tomato from this species $(7,57,61)$. Additionally, new TSWV isolates overcoming $S w-5$ have been identified in several parts of the world $(1,7,46,61)$. Latham et al. (25) deliberately developed two TSWV isolates, under controlled conditions, which completely infected plants homozygous for $S w-5$. Some of these TSWV isolates that infect $S w$ $5 / S w-5$ genotypes have been collected and used in this study.

In recognition of the paucity of comprehensive information on TSWV resistance in L. peruvianum and the identification of isolates that infect $S w-5 / S w-5$ plants, we initiated a screening of the $L$. peruvianum germplasm. In this study, 285 L. peruvianum accessions at the United States Department of Agriculture (USDA) Research Service, Cornell University Geneva, NY and the Tomato Genetics Resource Center (TGRC) at the University of California, Davis were screened for TSWV resistance to isolates completely overwhelming and partially overcoming $S w-5$.

\section{MATERIALS AND METHODS}

Management of Lycopersicon germplasm. In all, 169 TGRC accessions were screened, 38 of which belong to the core collection (henceforth identified as "TGRC Core") and 116 from the USDA collection, totaling $285 \mathrm{~L}$. peruvianum accessions (Tables 1 and 2). Seed of $L$. peruvianum and controls (near-isogenic tomato lines [NIL] 89R [Sw-5/Sw-5] and 89S [Sw- 
$\left.\left.5^{+} / S w-5^{+}\right]\right)$were scarified to increase germination with household bleach $(2.7 \%$ sodium hypochlorite) and distilled water at a 1:1 ratio for $30 \mathrm{~min}$., as recommended by Rick and Hunt (41). They then were washed three to four times with tap water and spread on moist germination paper (Anchor Paper Company, St. Paul, MN) in petri dishes. Germinated seedlings were transplanted to approximately $74 \mathrm{~cm}^{3} /$ plant cells filled with peat-based media in commercially available plastic flats. The objective was to test 50 plants per accession; however, actual numbers depended on germination and availability of seed. Eight cells of each flat were dedicated to $89 \mathrm{R}$ and 89S (four cells each) as a TSWV inoculation check. The 40 remaining cells were filled with one or more accessions depending on available seedlings.

Maintenance and use of TSWV isolates. Isolate TSWV6 was identified in Hawaii infecting $(S w-5 / S w-5)$ tomato plants (7). The anemone isolate $\left(\mathrm{An}_{\mathrm{wa}}-1\right)$, to which $S w-5$ provides some resistance, was identified on an Anemone sp. in Banjup, Western Australia in August 1998, and maintained in cv. Grosse Lisse $\left(\mathrm{S} w-5^{+} / \mathrm{S} w\right.$ $5^{+}$) (25). These isolates were selected based on three criteria. First, the isolates infected controls differently; TSWV6 infected both $S w-5$ and $S w-5^{+}$genotypes (7). However, $S w-5$ genotypes generally resisted $\mathrm{An}_{\mathrm{wa}}-1$ (25) but not $\mathrm{Sw}-5^{+}$genotypes. General symptoms of both TSWV6 and $\mathrm{An}_{\mathrm{wa}}-1$ were "tip blight" to wholeplant chlorosis and stunting in the controls, with $\mathrm{An}_{\mathrm{wa}}-1$ being the less aggressive of the two. Second, our experience suggested that both isolates produced reliable disease expression with our inoculation method. Finally, these two isolates were from different areas of the world.

Isolate maintenance and resistance screening trials were conducted in controlled access greenhouses at Brigham Young University, Provo, UT. Night temperatures ranged between approximately 18 and $25^{\circ} \mathrm{C}$ and day temperatures be- tween approximately 21 and $32^{\circ} \mathrm{C}$ throughout 10 months of screening.

Both isolates were maintained in Nicotiana rustica $\mathrm{L}$. and were transferred by rub inoculating Carborundum-dusted (600mesh) leaves with sterile cheesecloth pads dipped in 1 to $2^{\circ} \mathrm{C}$ buffer $(0.1 \mathrm{M}$ phosphate buffer, $\mathrm{pH}$ 7.4, $0.01 \mathrm{M}$ sodium sulfite), with homogenized infected tissue from both previously inoculated and systemically infected leaves. Isolates were transferred to young, healthy $N$. rustica every 10 days to maintain optimum virulence (57). Transfer of an isolate within 1 to 4 days after the appearance of visual symptoms on the inoculated $N$. rustica second to fourth true leaves produced the most reliable infection for mass inoculations $(6,54-$ 58; unpublished data).

Mass inoculation of accessions and controls were accomplished using techniques previously described $(54,57,58)$. Between 20 and 60 flats were inoculated in one setting. TSWV rapidly deactivates once inoculum is prepared; as a result, a set of up to 10 flats was inoculated within 12 to $15 \mathrm{~min}$, after which fresh inoculum was prepared for the next flat set. 89R and 89S TSWV checks were arranged such that two check plants were inoculated before and after every $10 \mathrm{~L}$. peruvianum plants. All plants were inoculated between their second and fourth true leaves and reinoculated with the same isolate 6 to 8 days later.

To monitor uncontrolled TSWV spread, 10 to 20 uninoculated $89 \mathrm{R}$ and $89 \mathrm{~S}$ plants were omnipresent in the greenhouse throughout the study. Utilizing mechanically transferred TSWV greatly reduces the possibility of unintended thrips transmission of this virus $(19,21)$. Additionally, a rotation of a regular set of insecticides (Maveric [Wellmark, Schaumburg, IL], Azatin XL [Olympic Horticultural Products, Mainland, PA], Conserve SC [Dow Agro Sciences, Indianapolis, IN], and Orthene 75 [Ortho, Marysville, $\mathrm{OH}$ ]) were used at labeled rates, approximately once a week, to minimize thrips and other arthro- pods. No instance of uncontrolled TSWV was identified throughout the study.

Evaluation of TSWV infection. Visual symptoms were used initially for eliminating obviously infected plants. Plants not showing characteristic TSWV symptoms were evaluated by peroxidase doubleantibody sandwich enzyme-linked immunosorbent assay (DAS-ELISA), supplied by Agdia Inc. (Elkhart, IN), to detect the presence or absence of the virus (57). Plants not responding in ELISA were maintained and visually reevaluated once a week for two additional months. We have observed (unpublished data) that plant stress often encourages phenotypic expression of symptomless TSWV carriers. Plants showing visual virus symptoms were eliminated and counted as infected. Consistently, those plants identified with optical density (OD) values near the threshold used by Stevens et al. (57) were found to become visually infected during the subsequent monitoring period.

Statistical analysis and ArcGIS program. SAS was used to develop the analysis of variance tables for statistical comparisons. The ArcGIS program (Environmental Systems Research Institute, Redlands, CA) was used to position the accessions to their geographical location according to accession passport information.

\section{RESULTS}

Summary of accessions response to TSWV isolates. Either the TSWV6 or $\mathrm{An}_{\mathrm{wa}}-1$ isolates were screened on a total of 16,335 L. peruvianum plants from 285 accessions, and 3,929 (1,899 89S and 2,030 89R) controls (Table 1). Of the 285 accessions screened for TSWV resistance, 172 (about 60\%) were tested with both isolates $(6,683$ and 5,516 L. peruvianum plants with TSWV6 and $\mathrm{An}_{\text {wa }}-1$, respectively; Table 2). No resistance was detected in 39 (about 23\%) of the 172 accessions to either TSWV isolate. Of the 172 accessions tested with both isolates, 23 (about $13 \%$ ) indicated resistance to $\mathrm{An}_{\mathrm{wa}}-1$ but

Table 1. Summary of Lycopersicon peruvianum Tomato spotted wilt virus (TSWV) resistance response to the Hawaiian (TSWV6) and Australian anemone $\left(\mathrm{An}_{\mathrm{wa}}-1\right)$ isolates across all accessions, individual plants, and L. esculentum $(89 \mathrm{~S}$ and $89 \mathrm{R})$ controls ${ }^{\mathrm{a}}$

\begin{tabular}{|c|c|c|c|c|c|c|c|c|c|c|c|c|}
\hline \multirow[b]{4}{*}{ Accessions } & \multicolumn{12}{|c|}{ TSWV isolates $^{\mathbf{b}}$} \\
\hline & \multicolumn{4}{|c|}{ Number of } & \multicolumn{8}{|c|}{ With resistant reaction } \\
\hline & \multicolumn{2}{|c|}{ Accessions tested } & \multicolumn{2}{|c|}{ Plants tested } & \multicolumn{2}{|c|}{ Plants (no.) } & \multicolumn{2}{|c|}{ Plants (\%) } & \multicolumn{2}{|c|}{ Accessions (no.) } & \multicolumn{2}{|c|}{ Accessions (\%) } \\
\hline & TSWV6 & $A n_{w a}-1$ & TSWV6 & $A n_{w a}-1$ & TSWV6 & $\mathrm{An}_{\mathrm{wa}}-1$ & TSWV6 & $A n_{w a}-1$ & TSWV6 & $A n_{w a}-1$ & TSWV6 & $\mathrm{An}_{\mathrm{wa}}-1$ \\
\hline TGRC Core & 35 & 24 & 767 & 962 & 24 & 51 & 3 & 5 & 12 & 10 & 34 & 42 \\
\hline TGRC & 129 & 111 & 5,559 & 3,575 & 497 & 426 & 9 & 12 & 81 & 66 & 63 & 57 \\
\hline USDA & 116 & 42 & 4,308 & 1,164 & 916 & 387 & 21 & 33 & 75 & 30 & 65 & 71 \\
\hline Totals & 280 & 177 & 10,634 & 5,701 & 1,437 & 864 & 14 & 15 & 168 & 106 & 60 & 59 \\
\hline \multicolumn{13}{|l|}{ Controls ${ }^{c}$} \\
\hline $89 \mathrm{~S}$ & $\ldots$ & $\ldots$ & 1,404 & 495 & 0 & 23 & 0 & 5 & $\ldots$ & $\ldots$ & $\ldots$ & $\ldots$ \\
\hline $89 \mathrm{R}$ & $\ldots$ & $\ldots$ & 1456 & 574 & 0 & 153 & 0 & 27 & $\ldots$ & $\ldots$ & $\ldots$ & $\ldots$ \\
\hline Totals & $\ldots$ & $\ldots$ & 2,860 & 1,069 & 0 & 176 & 0 & 16 & $\ldots$ & $\ldots$ & $\ldots$ & $\ldots$ \\
\hline
\end{tabular}

${ }^{a}$ In total, 285 L. peruvianum accessions were screened. Of those, 169 were from the Tomato Genetics Resource Center (TGRC), University of California, Davis, 38 of which belong to the core collection (TGRC Core), and 116 from the United States Department of Agriculture (USDA) Research Service collection located at the Cornell University Geneva, NY campus.

b TSWV isolate TSWV6 infects tomato with a $S w-5 / S w-5$ genotype and $\mathrm{An}_{\mathrm{wa}}-1$ partially infects the same genotype.

${ }^{c}$ Near-isogenic lines $89 \mathrm{~S}\left(S w-5^{+} / S w-5^{+}\right)$and $89 \mathrm{R}(S w-5 / S w-5)$ were used as controls. 
Table 2. All Lycopersicon peruvianum accessions tested and ranked according to average percentage of apparent resistant plants to Tomato spotted wilt virus (TSWV) isolates TSWV6 and anemone $\left(\mathrm{An}_{\mathrm{wa}}-1\right)^{\mathrm{a}}$

\begin{tabular}{|c|c|c|c|c|c|c|c|c|}
\hline \multirow[b]{3}{*}{ Accession no. } & \multicolumn{7}{|c|}{ Plants tested with TSWV isolates ${ }^{b}$} & \multirow[b]{3}{*}{ Origin of the accession ${ }^{c}$} \\
\hline & \multirow[b]{2}{*}{ Avg. (\%) } & \multicolumn{2}{|c|}{ Resistant (\%) } & \multicolumn{2}{|c|}{ Resistant (no.) } & \multicolumn{2}{|c|}{ Tested (no.) } & \\
\hline & & TSWV6 & $A n_{w a}-1$ & TSWV6 & $A n_{w a}-1$ & TSWV6 & $A n_{w a}-1$ & \\
\hline PI 126946 de & 68 & 72 & 64 & 33 & 23 & 46 & 36 & Culebras, Peru* \\
\hline PI $128648^{f}$ & 63 & 80 & 45 & 24 & 10 & 30 & 22 & Azapa Valley, Chile* \\
\hline LA 3636 & 63 & 63 & 63 & 19 & 27 & 30 & 43 & Coayllo, Lima, Peru** \\
\hline PI $128646^{\mathrm{f}}$ & 57 & 42 & 71 & 16 & 17 & 38 & 24 & Lluta/Arica, Chile* \\
\hline PI $128649^{f}$ & 57 & 43 & 70 & 17 & 19 & 40 & 27 & Arica, Chile* \\
\hline LA $2742^{\mathrm{f}}$ & 56 & 67 & 45 & 31 & 13 & 46 & 29 & Camarones-Guancarane, Tarapacá, Chile** \\
\hline PI $126930^{\text {de }}$ & 56 & 56 & 55 & 24 & 12 & 43 & 22 & Chancay, Peru* \\
\hline PI 127832 & 55 & 52 & 57 & 25 & 25 & 48 & 44 & Sacabaya, Peru* \\
\hline PI $128650^{\mathrm{f}}$ & 54 & 39 & 68 & 15 & 27 & 38 & 40 & Arica, Chile* \\
\hline PI $128652^{\text {fg }}$ & 54 & 48 & 60 & 23 & 25 & 48 & 42 & Arica, Chile* \\
\hline PI 128643 & 53 & 80 & 26 & 28 & 10 & 35 & 39 & Lima, Peru* \\
\hline LA 3900 & 52 & 67 & 36 & 32 & 16 & 48 & 45 & Canta, Lima, Peru** \\
\hline PI $126441^{\mathrm{h}}$ & 52 & 61 & 42 & 22 & 10 & 36 & 24 & Canta, Lima, Peru* \\
\hline PI 127831 & 51 & 55 & 46 & 21 & 19 & 38 & 41 & Sacabandía, Peru* \\
\hline PI $128645^{f i}$ & 49 & 55 & 43 & 26 & 16 & 47 & 37 & Lluta/Arica, Chile* \\
\hline PI $128647^{f}$ & 49 & 48 & 50 & 10 & 9 & 21 & 18 & Arica, Chile* \\
\hline PI 126945 & 49 & 36 & 62 & 12 & 23 & 33 & 37 & Culebras, Peru* \\
\hline LA 1677 & 48 & 23 & 73 & 5 & 30 & 22 & 41 & Fundo Huaquina to Topara, Lima, Peru** \\
\hline PI $126944^{\text {deghij }}$ & 48 & 39 & 57 & 11 & 21 & 28 & 37 & Culebras, Peru* \\
\hline PI $126928^{\text {dei }}$ & 47 & 54 & 39 & 26 & 19 & 48 & 49 & Pacasmayo, Peru* \\
\hline PI $126935^{\mathrm{h}}$ & 47 & 51 & 43 & 19 & 13 & 37 & 30 & Saña, Peru* \\
\hline LA 3640 & 41 & 54 & 28 & 26 & 10 & 48 & 36 & Mexico City, Mexico** \\
\hline LA $2745^{f}$ & 40 & 42 & 38 & 20 & 9 & 48 & 24 & Pan de Azúcar (Azapa), Tarapacá, Chile** \\
\hline LA 3858 & 39 & 40 & 38 & 19 & 15 & 48 & 40 & Canta, Lima, Peru** \\
\hline PI 127830 & 39 & 20 & 58 & 9 & 23 & 44 & 40 & Mollendo, Arequipa, Peru* \\
\hline LA $2770^{\text {f }}$ & 36 & 38 & 33 & 15 & 7 & 39 & 21 & Lluta, Tarapacá, Chile** \\
\hline G 30046 & 35 & 46 & 23 & 22 & 9 & 48 & 40 & Peru* \\
\hline LA 1975 & 33 & 28 & 37 & 13 & 10 & 47 & 27 & Desvio Santo Domingo, Lima, Peru** \\
\hline LA 2717 & 33 & 44 & 21 & 16 & 6 & 36 & 28 & Chilca, Lima, Peru** \\
\hline PI $128651^{\mathrm{f}}$ & 33 & 35 & 30 & 17 & 14 & 48 & 47 & Arica, Chile* \\
\hline LA 98 & 31 & 23 & 38 & 13 & 18 & 56 & 48 & Chilca, Lima, Peru** \\
\hline LA $2959^{f}$ & 31 & 31 & 31 & 13 & 12 & 42 & 39 & Chaca-Vitor, Tarapacá, Chile** \\
\hline PI 126926 & 31 & 44 & 18 & 21 & 5 & 48 & 28 & Pacasmayo, Peru* \\
\hline LA 2808 & 26 & 0 & 52 & 0 & 15 & 48 & 29 & Huaylas, Ancash, Peru** \\
\hline LA 3639 & 25 & 21 & 29 & 7 & 7 & 34 & 24 & Catac, Lima, Peru** \\
\hline LA 3799 & 25 & 38 & 11 & 18 & 4 & 47 & 37 & Rio Pativilca, Ancash, Peru** \\
\hline G 30027 & 25 & 48 & 2 & 23 & 1 & 48 & 43 & Peru* \\
\hline LA $2581^{f}$ & 24 & 22 & 26 & 9 & 5 & 41 & 19 & Chacarilla (4x), Tarapacá, Chile** \\
\hline LA 3637 & 24 & 19 & 28 & 9 & 8 & 48 & 29 & Coayllo, Lima, Peru** \\
\hline PI 326173 & 23 & 0 & 45 & 0 & 10 & 24 & 22 & South America* \\
\hline LA 1675 & 22 & 2 & 42 & 1 & 16 & 41 & 38 & Toparilla Cañón, Lima, Peru** \\
\hline PI 126929i & 22 & 0 & 44 & 0 & 11 & 13 & 25 & Pacasmayo, Peru* \\
\hline LA 1513 & 21 & 34 & 7 & 11 & 1 & 32 & 14 & Atiquipa, Arequipa, Peru** \\
\hline LA 1647 & 21 & 16 & 25 & 6 & 10 & 37 & 40 & Huaquina, Topara, Ica, Peru** \\
\hline LA 153 & 20 & 8 & 31 & 1 & 15 & 12 & 48 & Culebras, Ancash, Peru*** \\
\hline LA $464^{f}$ & 20 & 0 & 40 & 0 & 19 & 52 & 48 & Hacienda Rosario, Tarapacá, Chile** \\
\hline
\end{tabular}

\footnotetext{
a The TSWV6 isolate overcomes $S w-5 / S w-5$ genotypes where $\mathrm{An}_{\mathrm{wa}}-1$ partially overcomes the same genotype. In total, 285 . peruvianum accessions were screened. Of those, 169 were from the Tomato Genetics Resource Center (TGRC), University of California, Davis, 38 of which belong to the core collection (TGRC Core), and 116 from the United States Department of Agriculture (USDA) Research Service collection located at the Cornell University Geneva, NY campus.

b TSWV isolate TSWV6 infects $S w-5 / S w-5$ and $S w-5^{+} / S w-5$ genotypes and $\mathrm{An}_{\mathrm{wa}}-1$ genotypes partially infects these genotypes. Avg. $(\%)=$ percent average resistant.

c Asterisks *,**, and *** indicate collections from the USDA, TGRC, and TGRC Core, respectively.

d Eight L. peruvianum accessions that Smith and Paterson et al. reported on TSWV resistance in 1944 and 1989, respectively (34,52).

${ }^{\mathrm{e}}$ Nine accessions reported as being resistant to TSWV by Segeren et al. in $1993(49,50)$

$\mathrm{f}$ Twenty-six accessions from the southern tip of Peru and northern Chile, with the majority of the accessions associated with a high percentage of TSWV resistance (Fig. 1).

g Five L. peruvianum accessions identified by Cho et al. in 1996 (7) as being resistant to TSWV6.

${ }^{\text {h }}$ Five of the L. peruvianum accessions reported with TSWV resistance by Roselló et al. in 1999 (47).

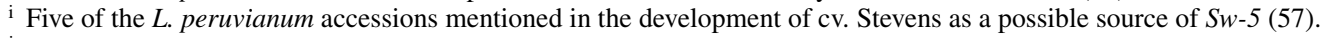

j L. peruvianum accession studied for TSWV resistance by Jordá et al. in 1993 (22).

${ }^{\mathrm{k}}$ L. peruvianum accession studied for TSWV resistance by Stevens et al. in 1994 (58).

${ }^{1}$ L. peruvianum accession studied and reported on for possible TSWV resistance by Boiteux et al. 1992 (4).

${ }^{\mathrm{m}}$ L. peruvianum accessions originating from Huallanca, Ancash, Peru, where PE-18 originated; PE-18 is the source of $S w-6$ (45-47).

${ }^{n}$ Three L. peruvianum accessions studied and reported as having a resistance response by Iizuka et al. in 1993 (20).

${ }^{\circ}$ L. peruvianum accession used by Gilbert and Tanaka in 1971 (15) for the development of 'Anahu' and also studied for TSWV resistance and reported on by Kumar et al. 1993 (24).

p L. peruvianum accession studied for TSWV resistance by Maluf et al. in 1991 (27).
} 
Table 2. (continued from preceding page)

\begin{tabular}{|c|c|c|c|c|c|c|c|c|}
\hline \multirow[b]{3}{*}{ Accession no. } & \multicolumn{7}{|c|}{ Plants tested with TSWV isolates ${ }^{\text {b }}$} & \multirow[b]{3}{*}{ Origin of the accession ${ }^{c}$} \\
\hline & \multirow[b]{2}{*}{ Avg. (\%) } & \multicolumn{2}{|c|}{ Resistant (\%) } & \multicolumn{2}{|c|}{ Resistant (no.) } & \multicolumn{2}{|c|}{ Tested (no.) } & \\
\hline & & TSWV6 & $A n_{w a}-1$ & TSWV6 & $\mathrm{An}_{\mathrm{wa}}-\mathbf{1}$ & TSWV6 & $A n_{w a}-1$ & \\
\hline LA 2962 & 20 & 19 & 21 & 9 & 6 & 47 & 29 & Chancay, Arequipa, Peru** \\
\hline LA $2744^{\mathrm{f}}$ & 18 & 9 & 26 & 3 & 12 & 32 & 47 & Sobraya (Azapa), Tarapacá, Chile*** \\
\hline LA 1373 & 17 & 10 & 24 & 4 & 9 & 40 & 37 & Asia, Lima, Peru** \\
\hline LA 1475 & 16 & 15 & 17 & 6 & 7 & 40 & 42 & Fundo "Los Anitos", Lima, Peru** \\
\hline LA 1692 & 16 & 12 & 19 & 3 & 5 & 25 & 27 & Putinza, Lima, Peru** \\
\hline PI $126444^{\mathrm{k}}$ & 16 & 23 & 9 & 11 & 4 & 48 & 43 & Canta, Peru* \\
\hline PI 306811 & 16 & 0 & 32 & 0 & 7 & 24 & 22 & United States* \\
\hline LA 444"gl & 15 & 14 & 15 & 3 & 7 & 22 & 47 & Chincha \#1, Ica, Peru*** \\
\hline LA 1300 & 15 & 0 & 29 & 0 & 14 & 53 & 48 & Santa Rosa de Quives, Lima, Peru** \\
\hline LA 2721 & 15 & 29 & 0 & 13 & 0 & 45 & 17 & Putinza, Lima, Peru** \\
\hline LA 2724 & 15 & 30 & 0 & 12 & 0 & 40 & 13 & Huaynilla, Lima, Peru** \\
\hline LA 1270 & 14 & 2 & 26 & 1 & 12 & 42 & 46 & Pisiquillo, Lima, Peru** \\
\hline LA 2068 & 14 & 27 & 0 & 10 & 0 & 37 & 27 & Chasquitambo, Ancash, Peru** \\
\hline LA $2732^{\mathrm{f}}$ & 14 & 8 & 19 & 1 & 9 & 13 & 48 & Moquella, Tarapaca, Chile*** \\
\hline LA 3795 & 14 & 28 & 0 & 13 & 0 & 46 & 53 & Alta Fortaleza, Ancash, Peru** \\
\hline LA 1473 & 12 & 19 & 5 & 9 & 2 & 48 & 39 & Callahuanca, Santa Eulalia, Lima, Peru** \\
\hline LA 1552 & 12 & 21 & 3 & 8 & 1 & 39 & 34 & San Mateo, Lima, Peru** \\
\hline LA $2562^{\mathrm{m}}$ & 12 & 5 & 19 & 2 & 4 & 42 & 21 & Huallanca, Ancash, Peru** \\
\hline LA 3797 & 12 & 18 & 6 & 7 & 2 & 39 & 34 & Anca Marca, Ancash. Peru** \\
\hline LA $111^{\mathrm{n}}$ & 11 & 1 & 21 & 1 & 10 & 122 & 48 & Zupe, Lima, Peru** \\
\hline LA 1616 & 11 & 0 & 21 & 0 & 3 & 13 & 14 & La Molina, La Rinconada, Lima, Peru** \\
\hline LA 1694 & 11 & 3 & 18 & 1 & 16 & 33 & 91 & Cacachuhuasin, Lima, Peru** \\
\hline PI 126439 & 11 & 22 & 0 & 10 & 0 & 46 & 17 & Canta, Peru* \\
\hline LA 1305 & 10 & 17 & 2 & 5 & 1 & 30 & 48 & Ticrapo, Huancavelica, Peru*** \\
\hline LA 1537 & 10 & 14 & 5 & 6 & 1 & 42 & 19 & Probably Peru** \\
\hline LA 1646 & 10 & 19 & 0 & 6 & 0 & 32 & 23 & Canta, Lima, Peru** \\
\hline LA 1358 & 9 & 13 & 5 & 4 & 2 & 31 & 41 & Yautan, Ancash, Peru** \\
\hline LA 1368 & 9 & 13 & 5 & 3 & 2 & 24 & 37 & San Jose de Palla, Lima, Peru** \\
\hline LA 1935 & 9 & 0 & 18 & 0 & 5 & 33 & 28 & Lomas de Atiquipa, Arequipa, Peru** \\
\hline LA 1133 & 9 & 0 & 17 & 0 & 8 & 58 & 48 & Huachipa, Lima, Peru** \\
\hline LA 2563 & 9 & 7 & 10 & 3 & 3 & 43 & 31 & Canon del Pato, Ancash, Peru** \\
\hline LA 2575 & 9 & 0 & 17 & 0 & 6 & 47 & 36 & Valle de Casma, Ancash, Peru** \\
\hline LA 2566 & 8 & 15 & 0 & 6 & 0 & 40 & 29 & Pomacocha-Llameyin, Rio Pocha, Ancash, Peru** \\
\hline LA 2573 & 8 & 2 & 14 & 1 & 5 & 48 & 36 & Valle de Casma, Ancash, Peru** \\
\hline G 30036 & 8 & 4 & 11 & 2 & 2 & 48 & 19 & Peru* \\
\hline LA 1292 & 7 & 8 & 5 & 1 & 1 & 13 & 22 & San Mateo, Lima, Peru*** \\
\hline LA $2561^{\mathrm{m}}$ & 7 & 3 & 10 & 1 & 2 & 39 & 20 & Huallanca, Ancash, Peru** \\
\hline LA 2331 & 7 & 13 & 0 & 6 & 0 & 47 & 43 & Agayapampa, La Libertad, Peru** \\
\hline LA 3664 & 7 & 14 & 0 & 1 & 0 & 7 & 13 & Nazca Grande, Ica, Peru** \\
\hline G 30040 & 7 & 13 & 0 & 6 & 0 & 48 & 20 & Peru* \\
\hline G 30044 & 7 & 0 & 13 & 0 & 1 & 42 & 8 & Peru* \\
\hline LA 1369 & 6 & 10 & 2 & 4 & 1 & 39 & 45 & San Gerónimo, Lima, Peru** \\
\hline LA 1377 & 6 & 5 & 6 & 1 & 2 & 20 & 36 & Navan, Lima, Peru** \\
\hline LA 1517 & 6 & 8 & 3 & 2 & 1 & 26 & 36 & Irrigación Santa Rosa, Lima, Peru** \\
\hline LA 1556 & 6 & 5 & 6 & 2 & 2 & 39 & 34 & Hacienda Higuereta, Lima, Peru** \\
\hline LA 2164 & 6 & 2 & 10 & 1 & 4 & 47 & 40 & Cuyca, Cajamarca, Peru** \\
\hline PI 127829 & 6 & 11 & 0 & 5 & 0 & 46 & 28 & San Juán/ Magdalena, Peru* \\
\hline G 30043 & 6 & 6 & 5 & 2 & 1 & 32 & 21 & Peru* \\
\hline LA 366 & 5 & 0 & 10 & 0 & 5 & 79 & 48 & Canta, Lima, Peru** \\
\hline LA 446 & 5 & 0 & 10 & 0 & 5 & 23 & 48 & Atiquipa, Arequipa, Peru** \\
\hline LA 1161 & 5 & 2 & 8 & 1 & 4 & 42 & 48 & Huachipa, Lima, Peru** \\
\hline LA 1626 & 5 & 10 & 0 & 2 & 0 & 21 & 27 & Mouth of Rio Rupac, Ancash, Peru*** \\
\hline LA 1977 & 5 & 9 & 0 & 4 & 0 & 47 & 23 & Orcocoto, Lima, Peru** \\
\hline LA 1981 & 5 & 6 & 3 & 3 & 1 & 48 & 40 & Vocatoma, Ancash, Peru** \\
\hline LA 2326 & 5 & 9 & 0 & 4 & 0 & 47 & 25 & Above Balsas, Amazonas, Peru** \\
\hline LA 2333 & 5 & 10 & 0 & 5 & 0 & 48 & 32 & Casmiche, La Libertad, Peru** \\
\hline LA 2388 & 5 & 2 & 8 & 1 & 3 & 48 & 39 & Cochabamba to Huambos, Cajamarca, Peru** \\
\hline LA 2555 & 5 & 9 & 0 & 3 & 0 & 34 & 41 & Mariscal Castilla, La Libertad, Peru** \\
\hline LA 3218 & 5 & 4 & 6 & 1 & 2 & 24 & 31 & Quebrada Guerrero, Arequipa, Peru** \\
\hline LA 3219 & 5 & 9 & 0 & 1 & 0 & 11 & 12 & Catarindo, Arequipa, Peru** \\
\hline LA 1554 & 4 & 8 & 0 & 3 & 0 & 36 & 24 & Huaral to Cerro de Pasco, Lima, Peru** \\
\hline LA 1913 & 4 & 3 & 5 & 1 & 1 & 39 & 22 & Tinguiyo, Ica, Peru** \\
\hline LA 2809 & 4 & 0 & 7 & 0 & 2 & 26 & 30 & Huaylas, Ancash, Peru** \\
\hline LA 2834 & 4 & 0 & 7 & 0 & 1 & 6 & 15 & Hacienda Asiento, Ica, Peru** \\
\hline LA 3154 & 4 & 2 & 6 & 1 & 2 & 48 & 34 & Otora-Puente Jahuay, Moquegua, Peru** \\
\hline LA 448 & 3 & 0 & 6 & 0 & 3 & 72 & 48 & Chala, Arequipa, Peru** \\
\hline LA 1294 & 3 & 5 & 0 & 1 & 0 & 19 & 47 & Surco, Lima, Peru** \\
\hline LA 1304 & 3 & 5 & 0 & 3 & 0 & 62 & 47 & Pámpano, Huancavelica, Peru** \\
\hline LA 1983 & 3 & 5 & 0 & 2 & 0 & 42 & 22 & Rio Manta, Ancash, Peru** \\
\hline LA 2327 & 3 & 6 & 0 & 3 & 0 & 48 & 33 & Aguas Calientes, Cajamarca, Peru** \\
\hline & & & & & & & & (continued on next page \\
\hline
\end{tabular}


Table 2. (continued from preceding page)

\begin{tabular}{|c|c|c|c|c|c|c|c|c|}
\hline \multirow[b]{3}{*}{ Accession no. } & \multicolumn{7}{|c|}{ Plants tested with TSWV isolates ${ }^{b}$} & \multirow[b]{3}{*}{ Origin of the accession ${ }^{c}$} \\
\hline & \multirow[b]{2}{*}{ Avg. (\%) } & \multicolumn{2}{|c|}{ Resistant (\%) } & \multicolumn{2}{|c|}{ Resistant (no.) } & \multicolumn{2}{|c|}{ Tested (no.) } & \\
\hline & & TSWV6 & $A n_{w a}-1$ & TSWV6 & $A n_{w a}-1$ & TSWV6 & $A n_{w a}-1$ & \\
\hline LA 2565 & 3 & 0 & 5 & 0 & 1 & 48 & 22 & Potrero de Pomacocha, Ancash, Peru** \\
\hline LA 3156 & 3 & 5 & 0 & 1 & 0 & 21 & 15 & Omate Valley, Moquegua, Peru** \\
\hline PI 127828 & 3 & 5 & 0 & 1 & 0 & 22 & 25 & San Juán, Cajamarca, Peru* \\
\hline G 30034 & 3 & 0 & 6 & 0 & 1 & 46 & 18 & Peru* \\
\hline LA 1337 & 2 & 1 & 2 & 1 & 1 & 81 & 48 & Atiquipa, Arequipa, Peru** \\
\hline LA 1379 & 2 & 0 & 4 & 0 & 2 & 36 & 47 & Cavajo, Lima, Peru*** \\
\hline LA 1653 & 2 & 0 & 3 & 0 & 1 & 36 & 37 & Uchumayo, Arequipa, Peru** \\
\hline LA 1937 & 2 & 4 & 0 & 1 & 0 & 27 & 8 & Quebrada Torrecillas, Arequipa, Peru*** \\
\hline LA 1949 & 2 & 3 & 0 & 1 & 0 & 34 & 12 & Las Calaveritas, Arequipa, Peru** \\
\hline LA 1951 & 2 & 3 & 0 & 1 & 0 & 32 & 39 & Ocoña, Arequipa, Peru*** \\
\hline LA 455 & 1 & 1 & 0 & 1 & 0 & 132 & 44 & Tambo, Arequipa, Peru** \\
\hline LA 752 & 1 & 0 & 2 & 0 & 1 & 48 & 48 & Sisacaya, Lima, Peru** \\
\hline LA 1280 & 1 & 1 & 0 & 1 & 0 & 102 & 48 & Chaciacayo, Lima, Peru** \\
\hline LA 1364 & 1 & 0 & 2 & 0 & 1 & 9 & 48 & Alta Fortaleza, Ancash, Peru*** \\
\hline LA 1551 & 1 & 2 & 0 & 1 & 0 & 42 & 19 & Surco, Lima, Peru** \\
\hline LA 1984 & 1 & 0 & 2 & 0 & 1 & 11 & 41 & Otuzco, La Libertad, Peru*** \\
\hline G 30039 & 1 & 2 & 0 & 1 & 0 & 41 & 12 & Peru* \\
\hline LA 378 & 0 & 0 & 0 & 0 & 0 & 110 & 47 & Cascas, Cajamarca, Peru** \\
\hline LA 451 & 0 & 0 & 0 & 0 & 0 & 36 & 47 & Arequipa, Arequipa, Peru** \\
\hline LA 453 & 0 & 0 & 0 & 0 & 0 & 57 & 46 & Yura, Arequipa, Peru** \\
\hline LA $462^{\text {ef }}$ & 0 & 0 & 0 & 0 & 0 & 60 & 35 & Sobraya, Tarapaca, Chile** \\
\hline LA 1032 & 0 & 0 & 0 & 0 & 0 & 27 & 47 & Aricampa, La Libertad, Peru** \\
\hline LA 1278 & 0 & 0 & 0 & 0 & 0 & 55 & 8 & Trapiche, Lima, Peru** \\
\hline LA 1331 & 0 & 0 & 0 & 0 & 0 & 21 & 40 & Nazca, Ica, Peru*** \\
\hline LA 1394 & 0 & 0 & 0 & 0 & 0 & 43 & 38 & Balsas, Amazonas, Peru** \\
\hline LA 1396 & 0 & 0 & 0 & 0 & 0 & 46 & 30 & Balsas (Chachapoyas), Amazonas, Peru** \\
\hline LA 1474 & 0 & 0 & 0 & 0 & 0 & 8 & 48 & Lomas, de Camaná, Arequipa, Peru*** \\
\hline LA 1708 & 0 & 0 & 0 & 0 & 0 & 39 & 9 & Chamaya to Jaen, Cajamarca, Peru** \\
\hline LA 1722 & 0 & 0 & 0 & 0 & 0 & 18 & 22 & Ticrapo Viejo, Huancavelica, Peru** \\
\hline LA 1723 & 0 & 0 & 0 & 0 & 0 & 3 & 3 & La Quinga, Ica, Peru** \\
\hline LA 1744 & 0 & 0 & 0 & 0 & 0 & 42 & 20 & Putinza, Lima, Peru** \\
\hline LA 1910 & 0 & 0 & 0 & 0 & 0 & 36 & 3 & Tambillo, Huancavelica, Peru*** \\
\hline LA 1929 & 0 & 0 & 0 & 0 & 0 & 13 & 48 & La Yapana, Ica, Peru*** \\
\hline LA 1944 & 0 & 0 & 0 & 0 & 0 & 41 & 19 & Rio Atico, Arequipa, Peru** \\
\hline LA 1945 & 0 & 0 & 0 & 0 & 0 & 7 & 36 & Caravelí, Arequipa, Peru*** \\
\hline LA 1947 & 0 & 0 & 0 & 0 & 0 & 21 & 21 & Puerto Atico, Arequipa, Peru** \\
\hline LA 1955 & 0 & 0 & 0 & 0 & 0 & 46 & 24 & Matarani, Arequipa, Peru** \\
\hline LA 1985 & 0 & 0 & 0 & 0 & 0 & 47 & 35 & Casmiche, La Libertad, Peru** \\
\hline LA 2163 & 0 & 0 & 0 & 0 & 0 & 45 & 45 & Cochabamba to Yamaluc, Cajamarca, Peru*** \\
\hline LA 2185 & 0 & 0 & 0 & 0 & 0 & 37 & 44 & Pongo de Rentema, Amazonas, Peru*** \\
\hline LA 2330 & 0 & 0 & 0 & 0 & 0 & 45 & 20 & Chagual, La Libertad, Peru** \\
\hline LA 2964 & 0 & 0 & 0 & 0 & 0 & 49 & 48 & Quebrada de Burros, Tacna, Peru*** \\
\hline LA 2981-B & 0 & 0 & 0 & 0 & 0 & 47 & 47 & Torata to Chilligua, Moquegua, Peru*** \\
\hline LA 3220 & 0 & 0 & 0 & 0 & 0 & 5 & 15 & Cocachacra-Quebrada Cachendo, Arequipa, Peru** \\
\hline LA 3666 & 0 & 0 & 0 & 0 & 0 & 1 & 24 & La Yapa, Ica, Peru** \\
\hline LA 3781 & 0 & 0 & 0 & 0 & 0 & 7 & 27 & Quebrada Oscollo, Arequipa, Peru** \\
\hline LA 3783 & 0 & 0 & 0 & 0 & 0 & 11 & 16 & Chapana, Arequipa, Peru** \\
\hline LA 3787 & 0 & 0 & 0 & 0 & 0 & 26 & 21 & Alta Chaparra, Arequipa, Peru** \\
\hline LA 3790 & 0 & 0 & 0 & 0 & 0 & 16 & 33 & Caravelí, Arequipa, Peru** \\
\hline G 30028 & 0 & 0 & 0 & 0 & 0 & 47 & 25 & Peru* \\
\hline G 30029 & 0 & 0 & 0 & 0 & 0 & 34 & 19 & Peru* \\
\hline G 30032 & 0 & 0 & 0 & 0 & 0 & 45 & 22 & Peru* \\
\hline G 30033 & 0 & 0 & 0 & 0 & 0 & 46 & 23 & Peru* \\
\hline G 30035 & 0 & 0 & 0 & 0 & 0 & 44 & 8 & Peru* \\
\hline G 30037 & 0 & 0 & 0 & 0 & 0 & 30 & 13 & Peru* \\
\hline G 30045 & 0 & 0 & 0 & 0 & 0 & 48 & 7 & Peru* \\
\hline PI 251307 & $\ldots$ & 74 & $\ldots$ & 25 & $\ldots$ & 34 & 0 & Cascas/La Libertad, Peru* \\
\hline PI $128660^{\mathrm{dfg}}$ & $\ldots$ & 60 & $\ldots$ & 28 & $\ldots$ & 47 & 0 & Tacna, Peru* \\
\hline PI 251306 & $\ldots$ & 59 & $\ldots$ & 23 & $\ldots$ & 39 & 0 & Culebras/Ancash, Peru* \\
\hline PI $143679^{\mathrm{h}}$ & $\ldots$ & 54 & $\ldots$ & 25 & $\ldots$ & 46 & 0 & Manabi, Ecuador* \\
\hline PI $128653^{\mathrm{f}}$ & $\ldots$ & 53 & $\ldots$ & 24 & $\ldots$ & 45 & 0 & Arica, Chile* \\
\hline PI 129147 & $\ldots$ & 48 & $\ldots$ & 23 & $\ldots$ & 48 & 0 & Guayaquil/Salinas, Ecuador* \\
\hline PI 129152 & $\ldots$ & 46 & $\ldots$ & 22 & $\ldots$ & 48 & 0 & Guayaquil, Ecuador* \\
\hline PI 129145 & $\ldots$ & 45 & $\ldots$ & 20 & $\ldots$ & 44 & 0 & Tambo, Peru* \\
\hline PI $128654^{\mathrm{fi}}$ & $\ldots$ & 44 & $\ldots$ & 14 & $\ldots$ & 32 & 0 & Arica, Chile* \\
\hline PI $128656^{\mathrm{f}}$ & $\ldots$ & 41 & $\ldots$ & 12 & $\ldots$ & 29 & 0 & Characarilla/Tarapaca, Chile* \\
\hline PI $128657^{\text {defo }}$ & $\ldots$ & 40 & $\ldots$ & 19 & $\ldots$ & 47 & 0 & Tacna, Peru* \\
\hline PI 246586 97GI & $\ldots$ & 39 & $\ldots$ & 16 & $\ldots$ & 41 & 0 & Atico/Arequipa, Peru* \\
\hline \multirow[t]{2}{*}{ PI $128655^{\mathrm{f}}$} & $\ldots$ & 38 & $\ldots$ & 10 & $\ldots$ & 26 & 0 & Chacarilla/Tarapaca, Chile* \\
\hline & & & & & & & & (continued on next page) \\
\hline
\end{tabular}


Table 2. (continued from preceding page)

\begin{tabular}{|c|c|c|c|c|c|c|c|c|}
\hline \multirow[b]{3}{*}{ Accession no. } & \multicolumn{7}{|c|}{ Plants tested with TSWV isolates ${ }^{b}$} & \multirow[b]{3}{*}{ Origin of the accession ${ }^{c}$} \\
\hline & \multirow[b]{2}{*}{ Avg. (\%) } & \multicolumn{2}{|c|}{ Resistant (\%) } & \multicolumn{2}{|c|}{ Resistant (no.) } & \multicolumn{2}{|c|}{ Tested (no.) } & \\
\hline & & TSWV6 & $\mathrm{An}_{\mathrm{wa}}-\mathbf{1}$ & TSWV6 & $A n_{w a}-1$ & TSWV6 & $A n_{w a}-1$ & \\
\hline PI 128663 & $\ldots$ & 35 & $\ldots$ & 17 & $\ldots$ & 48 & 0 & Moquegua, Peru* \\
\hline PI 129149 & $\ldots$ & 33 & $\ldots$ & 10 & $\ldots$ & 30 & 0 & Guayaquil, Ecuador* \\
\hline PI 390671 & $\ldots$ & 32 & $\ldots$ & 9 & $\ldots$ & 28 & 0 & Lima, Peru* \\
\hline PI 270435 & $\ldots$ & 30 & $\ldots$ & 14 & $\ldots$ & 47 & 0 & Mexico City, Mexico* \\
\hline PI $251311^{\mathrm{h}}$ & $\ldots$ & 25 & $\ldots$ & 10 & $\ldots$ & 40 & 0 & Arequipa, Peru* \\
\hline PI 251308 & $\ldots$ & 24 & $\ldots$ & 7 & $\ldots$ & 29 & 0 & Atiquipa/Arequipa, Peru* \\
\hline PI $128661^{\mathrm{f}}$ & $\ldots$ & 23 & $\ldots$ & 10 & $\ldots$ & 43 & 0 & Tacna, Peru* \\
\hline PI 390682 & $\ldots$ & 23 & $\ldots$ & 11 & $\ldots$ & 48 & 0 & Lima, Peru* \\
\hline PI 390681 & $\ldots$ & 21 & $\ldots$ & 6 & $\ldots$ & 28 & 0 & Lima, Peru* \\
\hline LA 1609 & $\ldots$ & 20 & $\ldots$ & 2 & $\ldots$ & 10 & 0 & Asia-El Pinon, Lima, Peru*** \\
\hline PI 251309 & $\ldots$ & 19 & $\ldots$ & 8 & $\ldots$ & 42 & 0 & Chala/Arequipa, Peru* \\
\hline PI 251310 & $\ldots$ & 19 & $\ldots$ & 4 & $\ldots$ & 21 & 0 & Atico, Peru* \\
\hline PI 266375 & $\ldots$ & 19 & $\ldots$ & 5 & $\ldots$ & 26 & 0 & Lima, Peru* \\
\hline PI 390672 & $\ldots$ & 18 & $\ldots$ & 8 & $\ldots$ & 44 & 0 & Lima, Peru* \\
\hline PI 390670 & $\ldots$ & 17 & $\ldots$ & 7 & $\ldots$ & 42 & 0 & Lima, Peru* \\
\hline PI 246586 60OI & $\ldots$ & 16 & $\ldots$ & 5 & $\ldots$ & 31 & 0 & Atico/Arequipa, Peru* \\
\hline PI $128659^{\text {dfg }}$ & $\ldots$ & 13 & $\ldots$ & 6 & $\ldots$ & 45 & 0 & Tacna, Peru* \\
\hline LA 1365 & $\ldots$ & 12 & $\ldots$ & 3 & $\ldots$ & 25 & 0 & Caranquillo, Ancash, Peru*** \\
\hline PI $129146^{\mathrm{de}}$ & $\ldots$ & 12 & $\ldots$ & 2 & $\ldots$ & 17 & 0 & Tambo, Peru* \\
\hline PI 365956 & $\ldots$ & 12 & $\ldots$ & 3 & $\ldots$ & 25 & 0 & Lima, Peru* \\
\hline PI $128658^{\mathrm{f}}$ & $\ldots$ & 11 & $\ldots$ & 5 & $\ldots$ & 45 & 0 & Tacna, Peru* \\
\hline PI 365942 & $\ldots$ & 11 & $\ldots$ & 2 & $\ldots$ & 18 & 0 & Lima, Peru* \\
\hline PI 246585 & $\ldots$ & 9 & $\ldots$ & 3 & $\ldots$ & 32 & 0 & Ayacucho, Peru* \\
\hline PI 390684 & $\ldots$ & 8 & $\ldots$ & 3 & $\ldots$ & 36 & 0 & Lima, Peru* \\
\hline LA 1350 & $\ldots$ & 8 & $\ldots$ & 1 & $\ldots$ & 12 & 0 & Chauna, Cajamarca, Peru** \\
\hline LA 110 & $\ldots$ & 7 & $\ldots$ & 9 & $\ldots$ & 123 & 0 & Cajacay, Ancash, Peru** \\
\hline PI 379029 & $\ldots$ & 7 & $\ldots$ & 3 & $\ldots$ & 44 & 0 & Tambo, Peru* \\
\hline PI 390680 & $\ldots$ & 7 & $\ldots$ & 3 & $\ldots$ & 43 & 0 & Lima, Peru* \\
\hline LA 445 & $\ldots$ & 6 & $\ldots$ & 3 & $\ldots$ & 51 & 0 & Chincha \#2, Ica, Peru** \\
\hline PI 390664 & $\ldots$ & 6 & $\ldots$ & 2 & $\ldots$ & 32 & 0 & Paramonga, Peru* \\
\hline LA 364 & $\ldots$ & 5 & $\ldots$ & 1 & $\ldots$ & 22 & 0 & Canta, Lima, Peru** \\
\hline LA 1293 & $\ldots$ & 5 & $\ldots$ & 2 & $\ldots$ & 43 & 0 & Matucana, Lima, Peru** \\
\hline PI 303814 & $\ldots$ & 5 & $\ldots$ & 2 & $\ldots$ & 43 & 0 & Idaho, United States* \\
\hline PI 365946 & $\ldots$ & 5 & $\ldots$ & 1 & $\ldots$ & 20 & 0 & Puquino, Peru* \\
\hline LA 370 & $\ldots$ & 4 & $\ldots$ & 3 & $\ldots$ & 79 & 0 & Hacienda Huampaní, Lima, Peru** \\
\hline PI 365943 & $\ldots$ & 4 & $\ldots$ & 2 & $\ldots$ & 48 & 0 & Lima, Peru* \\
\hline PI 390669 & $\ldots$ & 4 & $\ldots$ & 2 & $\ldots$ & 48 & 0 & Lima, Peru* \\
\hline LA $372^{\text {n }}$ & $\ldots$ & 3 & $\ldots$ & 3 & $\ldots$ & 91 & 0 & Culebras \#1, Ancash, Peru** \\
\hline LA 1271 & $\ldots$ & 3 & $\ldots$ & 1 & $\ldots$ & 37 & 0 & Horcón, Lima, Peru** \\
\hline PI 379015 & $\ldots$ & 3 & $\ldots$ & 1 & $\ldots$ & 40 & 0 & Ticrapo, Peru* \\
\hline LA 392 & $\ldots$ & 2 & $\ldots$ & 1 & $\ldots$ & 45 & 0 & Llallan, Cajamarca, Peru** \\
\hline LA $1982^{\mathrm{m}}$ & $\ldots$ & 2 & $\ldots$ & 1 & $\ldots$ & 42 & 0 & Huallanca, Ancash, Peru*** \\
\hline LA 1283 & $\ldots$ & 2 & $\ldots$ & 1 & $\ldots$ & 41 & 0 & Sta. Cruz de Laya, Lima, Peru** \\
\hline PI 365947 & $\ldots$ & 2 & $\ldots$ & 1 & $\ldots$ & 47 & 0 & Arequipa, Peru* \\
\hline PI 365950 & $\ldots$ & 2 & $\ldots$ & 1 & $\ldots$ & 48 & 0 & Caciche, Peru* \\
\hline LA $371^{\mathrm{e}}$ & $\ldots$ & 0 & $\ldots$ & 0 & $\ldots$ & 79 & 0 & Zupe, Lima, Peru** \\
\hline LA 374 & $\ldots$ & 0 & $\ldots$ & 0 & $\ldots$ & 107 & 0 & Culebras \#2, Ancash, Peru** \\
\hline LA $441^{p}$ & $\ldots$ & 0 & $\ldots$ & 0 & $\ldots$ & 3 & 0 & Cerro Campana, La Libertad, Peru*** \\
\hline LA 454 & $\ldots$ & 0 & $\ldots$ & 0 & $\ldots$ & 15 & 0 & Tambo, Arequipa, Peru*** \\
\hline LA 1027 & $\ldots$ & 0 & $\ldots$ & 0 & $\ldots$ & 13 & 0 & Cajamarca, Peru** \\
\hline LA 1031 & $\ldots$ & 0 & $\ldots$ & 0 & $\ldots$ & 92 & 0 & Balsas, Amazonas, Peru** \\
\hline LA 1274 & $\ldots$ & 0 & $\ldots$ & 0 & $\ldots$ & 2 & 0 & Pocabamba, Lima, Peru*** \\
\hline LA 1284 & $\ldots$ & 0 & $\ldots$ & 0 & $\ldots$ & 30 & 0 & Espiritu Santo, Lima, Peru** \\
\hline LA 1296 & $\ldots$ & 0 & $\ldots$ & 0 & $\ldots$ & 8 & 0 & Tornamesa, Lima, Peru** \\
\hline LA 1333 & $\ldots$ & 0 & $\ldots$ & 0 & $\ldots$ & 76 & 0 & Loma Camana, Arequipa, Peru** \\
\hline LA 1336 & $\ldots$ & 0 & $\ldots$ & 0 & $\ldots$ & 39 & 0 & Atico, Arequipa, Peru** \\
\hline LA 1339 & $\ldots$ & 0 & $\ldots$ & 0 & $\ldots$ & 35 & 0 & Capillucas, Lima, Peru*** \\
\hline LA 1346 & $\ldots$ & 0 & $\ldots$ & 0 & $\ldots$ & 51 & 0 & Caciche, La Libertad, Peru** \\
\hline LA 1351 & $\ldots$ & 0 & $\ldots$ & 0 & $\ldots$ & 6 & 0 & Rupe, Cajamarca, Peru*** \\
\hline LA 1360 & $\ldots$ & 0 & $\ldots$ & 0 & $\ldots$ & 29 & 0 & Pariacoto, Ancash, Peru*** \\
\hline LA 1395 & $\ldots$ & 0 & $\ldots$ & 0 & $\ldots$ & 26 & 0 & Chachapoyas, Amazonas, Peru*** \\
\hline LA 1973 & $\ldots$ & 0 & $\ldots$ & 0 & $\ldots$ & 15 & 0 & Yura, Arequipa, Peru*** \\
\hline LA 2152 & $\ldots$ & 0 & $\ldots$ & 0 & $\ldots$ & 2 & 0 & Cospán, Cajamarca, Peru**** \\
\hline LA 2172 & $\ldots$ & 0 & $\ldots$ & 0 & $\ldots$ & 35 & 0 & Cuyca, Cajamarca, Peru*** \\
\hline LA 2328 & $\ldots$ & 0 & $\ldots$ & 0 & $\ldots$ & 1 & 0 & Aricapampa, La Libertad, Peru*** \\
\hline LA 3853 & $\ldots$ & 0 & $\ldots$ & 0 & $\ldots$ & 22 & 0 & Moyopampa, La Libertad, Peru** \\
\hline PI 251314 & $\ldots$ & 0 & $\ldots$ & 0 & $\ldots$ & 24 & 0 & The Rio Jequetepeque, Peru* \\
\hline PI 266376 & $\ldots$ & 0 & $\ldots$ & 0 & $\ldots$ & 14 & 0 & Magdalena/Cajamarca, Peru* \\
\hline PI 365938 & $\ldots$ & 0 & $\ldots$ & 0 & $\ldots$ & 15 & 0 & Pocabamba/Lima, Peru* \\
\hline \multirow[t]{2}{*}{ PI 365939} & $\ldots$ & 0 & $\ldots$ & 0 & $\ldots$ & 31 & 0 & Pocabamba, Peru* \\
\hline & & & & & & & & (continued on next page) \\
\hline
\end{tabular}


Table 2. (continued from preceding page)

\begin{tabular}{|c|c|c|c|c|c|c|c|c|}
\hline \multirow[b]{3}{*}{ Accession no. } & \multicolumn{7}{|c|}{ Plants tested with TSWV isolates ${ }^{b}$} & \multirow[b]{3}{*}{ Origin of the accession ${ }^{c}$} \\
\hline & \multirow[b]{2}{*}{ Avg. (\%) } & \multicolumn{2}{|c|}{ Resistant (\%) } & \multicolumn{2}{|c|}{ Resistant (no.) } & \multicolumn{2}{|c|}{ Tested (no.) } & \\
\hline & & TSWV6 & $\mathrm{An}_{\mathrm{wa}} \mathbf{- 1}$ & TSWV6 & $\mathrm{An}_{\mathrm{wa}} \mathbf{- 1}$ & TSWV6 & $\mathrm{An}_{\mathrm{wa}} \mathbf{- 1}$ & \\
\hline PI 365940 & $\ldots$ & 0 & $\ldots$ & 0 & $\ldots$ & 23 & 0 & Pocabamba, Peru* \\
\hline PI 365941 & $\ldots$ & 0 & $\ldots$ & 0 & $\ldots$ & 4 & 0 & Quilca/Lima, Peru* \\
\hline PI 365948 & $\ldots$ & 0 & $\ldots$ & 0 & $\ldots$ & 45 & 0 & Carretera, Peru* \\
\hline PI 365951 & $\ldots$ & 0 & $\ldots$ & 0 & $\ldots$ & 17 & 0 & Contumazá, Peru* \\
\hline PI 365952 & $\ldots$ & 0 & $\ldots$ & 0 & $\ldots$ & 13 & 0 & Huaras, Peru* \\
\hline PI 365953 & $\ldots$ & 0 & $\ldots$ & 0 & $\ldots$ & 24 & 0 & Caranquillo, Peru* \\
\hline PI 365955 & $\ldots$ & 0 & $\ldots$ & 0 & $\ldots$ & 17 & 0 & Lima, Peru* \\
\hline PI 365968 & $\ldots$ & 0 & $\ldots$ & 0 & $\ldots$ & 30 & 0 & Cuzco, Peru* \\
\hline PI 365969 & $\ldots$ & 0 & $\ldots$ & 0 & $\ldots$ & 33 & 0 & Yaca, Peru* \\
\hline PI 379016 & $\ldots$ & 0 & $\ldots$ & 0 & $\ldots$ & 30 & 0 & Chauna, Peru* \\
\hline PI 379017 & $\ldots$ & 0 & $\ldots$ & 0 & $\ldots$ & 15 & 0 & Lima, Peru* \\
\hline PI 379018 & $\ldots$ & 0 & $\ldots$ & 0 & $\ldots$ & 48 & 0 & Lima, Peru* \\
\hline PI 390665 & $\ldots$ & 0 & $\ldots$ & 0 & $\ldots$ & 48 & 0 & Camaná, Peru* \\
\hline PI 390666 & $\ldots$ & 0 & $\ldots$ & 0 & $\ldots$ & 44 & 0 & Lima, Peru* \\
\hline PI 390667 & $\ldots$ & 0 & $\ldots$ & 0 & $\ldots$ & 47 & 0 & Lima, Peru* \\
\hline PI 390668 & $\ldots$ & 0 & $\ldots$ & 0 & $\ldots$ & 24 & 0 & Lima, Peru* \\
\hline PI 390673 & $\ldots$ & 0 & $\ldots$ & 0 & $\ldots$ & 42 & 0 & Lima, Peru* \\
\hline PI 390674 & $\ldots$ & 0 & $\ldots$ & 0 & $\ldots$ & 42 & 0 & Lima, Peru* \\
\hline PI 390675 & $\ldots$ & 0 & $\ldots$ & 0 & $\ldots$ & 48 & 0 & Lima, Peru* \\
\hline PI 390676 & $\ldots$ & 0 & $\ldots$ & 0 & $\ldots$ & 48 & 0 & Lima, Peru* \\
\hline PI 390678 & $\ldots$ & 0 & $\ldots$ & 0 & $\ldots$ & 48 & 0 & Lima, Peru* \\
\hline PI 390679 & $\ldots$ & 0 & $\ldots$ & 0 & $\ldots$ & 48 & 0 & Lima, Peru* \\
\hline PI 390683 & $\ldots$ & 0 & $\ldots$ & 0 & $\ldots$ & 48 & 0 & La Libertad, Peru* \\
\hline PI 390685 & $\ldots$ & 0 & $\ldots$ & 0 & $\ldots$ & 48 & 0 & Cerro de Pasco, Peru* \\
\hline PI 390687 & $\ldots$ & 0 & $\ldots$ & 0 & $\ldots$ & 22 & 0 & Canchaque, Peru* \\
\hline LA 107 & $\ldots$ & $\ldots$ & 4 & $\ldots$ & 2 & 0 & 47 & Hacienda San Isidro, Lima, Peru*** \\
\hline LA $385^{n}$ & $\ldots$ & $\ldots$ & 0 & $\ldots$ & 0 & 0 & 48 & San Juán, Cajamarca, Peru** \\
\hline LA 1989 & $\ldots$ & $\ldots$ & 0 & $\ldots$ & 0 & 0 & 4 & $* *$ \\
\hline LA 2157 & $\ldots$ & $\ldots$ & 0 & $\ldots$ & 0 & 0 & 38 & Tunel Chotano, Cajamarca, Peru*** \\
\hline LA 2553 & $\ldots$ & $\ldots$ & 0 & $\ldots$ & 0 & 0 & 48 & Balconcillo de San Marcos, Cajamarca, Peru*** \\
\hline
\end{tabular}

not to TSWV6 and 31 (about 18\%) had plants with a resistance response to TSWV6 and not to $\mathrm{An}_{\mathrm{wa}}-1$. In all, 79 accessions had plants indicating resistance to both isolates (Table 2).

A distinct difference in the TSWV resistance response was identified between germplasm collections. The USDA and TGRC collections had a significantly $(P<$ 0.0001 ) higher level of resistance than the TGRC Core collection. Of the 172 accessions tested with both isolates, 35 of 42 (approximately 83\%) USDA, 86 of 109 (approximately 79\%) TGRC, and 12 of 21 (approximately 57\%) TGRC Core indicated resistance to at least one of the two isolates (Table 2).

Germplasm responses to TSWV6 and $\mathbf{A n}_{\mathbf{w a}} \mathbf{- 1}$. When inoculated with TSWV6, all $1,40489 \mathrm{~S}$ and $1,45689 \mathrm{R}$ controls were infected (Table 1). Of the 10,634 L. peruvianum plants tested from 280 accessions, $14 \%$ of the plants were found to be virus free, which was significantly higher than the controls. The 116 USDA, 129 TGRC, and 35 TGRC Core accessions tested with TSWV6 were significantly different from each other $(P$ value $<0.0001)$ with 21,9 , and $3 \%$ of the plants, respectively, virus free (Table 1; Fig. 1A). This pattern was similar when comparing accessions with plants free of the virus (Table 1; Fig. 1B).

$\mathrm{An}_{\text {wa }}-1$ was less virulent than TSWV6; $95 \%$ of the $49589 \mathrm{~S}$ and $73 \%$ of the 574 $89 \mathrm{R}$ controls were infected (Table 1). Of the 5,705 L. peruvianum tested with $\mathrm{An}_{\text {wa }}{ }^{-}$
1, $15 \%$ indicated resistance, and these plants were found within 106 of the 177 (59\%) accessions tested (Table 1). Using $\mathrm{An}_{\text {wa }}-1$, the 42 USDA, 111 TGRC, and 24 TGRC Core accessions were found to be significantly different from each other $(\mathrm{P}$ value $<0.0001$ ) with 33,12 , and $5 \%$ of the plants, respectively, virus free (Table 1; Fig. 1B).

Geographic distribution of $L$. peruvianum TSWV resistance. We were able to examine the geographical distribution of 260 L. peruvianum accessions with areaspecific passport data. Of those 260 accessions, 154 were tested with both TSWV isolates, 256 were tested with TSWV6 (Fig. 2A; Table 2) and 158 with $\mathrm{An}_{\text {wa }}-1$ (Fig. 2B; Table 2). Resistance to both TSWV isolates was somewhat equally distributed throughout Peru, with perhaps more emphasis along the Peruvian Pacific coastal plane (Fig. 2). However, the Arica, Chile and southern Peru region indicated an unusually high ratio of accessions with resistant plants (Fig. 2, insert 2). Specifically, 23 of 26 (approximately 88\%) and 16 of 17 (approximately 94\%) of the accessions had $>8 \%$ of their plants resistant to TSWV6 and $\mathrm{An}_{\mathrm{wa}}-1$, respectively (Fig. 2 ). This region can be compared with the insert 1 area of Figure 2A and 2B (surrounding Lima, Peru) in which 45 of 102 (about 44\%) and 29 of 58 (about 50\%) accessions had $>8 \%$ of the plants, indicating a resistance response to TSWV6 and $\mathrm{An}_{\mathrm{wa}}-1$, respectively.
There is an even greater difference in the accessions from the Arica region (Fig. 2 , insert 2), which had $\geq 33 \%$ of their plants indicate a resistant response. In all, 19 of 26 (about $73 \%$ ) and 12 of 17 (about $71 \%$ ) accessions had a resistance response to TSWV6 and $\mathrm{An}_{\mathrm{wa}}-1$, respectively (Fig. 2 , insert 2) compared with the region around Lima (Fig. 2, insert 1), where 12 accessions tested with either TSWV6 (about $12 \%$ ) or $\mathrm{An}_{\mathrm{wa}}-1$ (about $21 \%$ ) had $\geq 33 \%$ of their plants free of TSWV.

\section{DISCUSSION}

Mechanical inoculation. Several reports have utilized thrips for screening smaller germplasm populations for TSWV resistance $(24,44,47)$. However, due to the impracticalities of managing or controlling thrips, we utilized mechanical inoculation to screen our large plant numbers with two TSWV isolates. Especially when some plants or accessions could be considered TSWV resistant when, in reality, thrips could be ether deterred (preference) or possibly resisted by some $L$. peruvianum accessions (24).

A noted drawback to serial mechanical inoculation has been the generation of defective interfering RNAs (DI RNAs) causing attenuation of the TSWV isolate. Generation of these defective isolates can be aggravated during some environmental regimes $(19,21,39,40)$. Nonetheless, there are usable stable TSWV isolates with DI RNAs. Ie (19) described TSWV isolates 
A

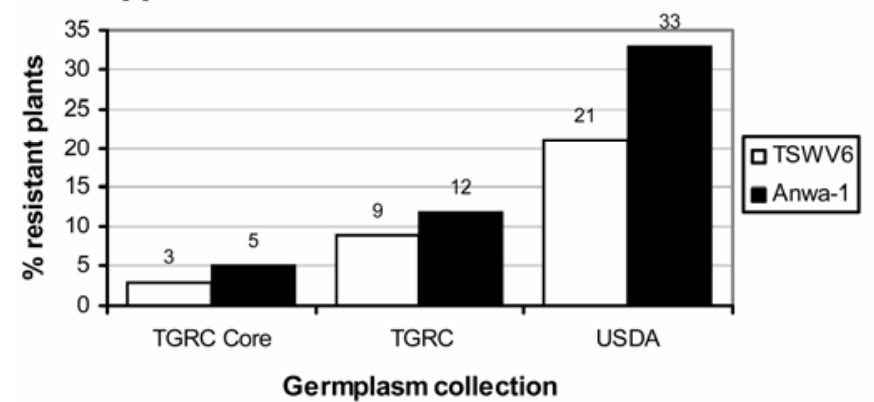

B

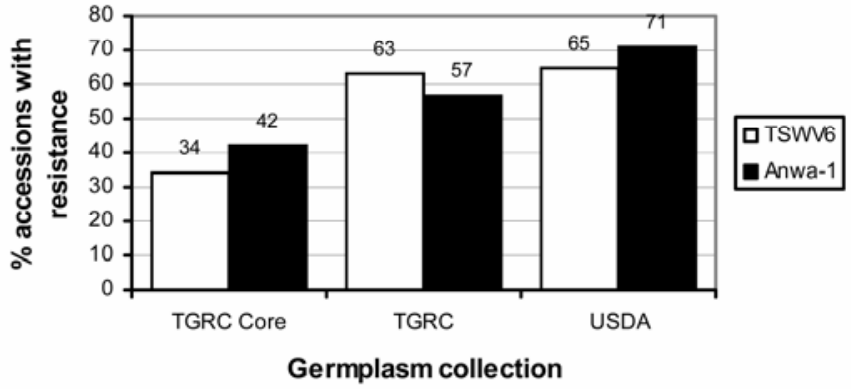

Fig. 1. Tomato spotted wilt virus (TSWV) resistance response of the accessions from the United States Department of Agriculture (USDA) Research Service located at the Cornell University Geneva, NY campus, Tomato Genetics Resource Center (TGRC), University of California, Davis, and TGRC-Core collections to the Hawaiian isolate (TSWV 6) and to the anemone isolate $\left(\mathrm{An}_{\mathrm{wa}}-1\right)$ expressed as a percentage of plants free of infection.

\section{A TSWV6 Isolate}

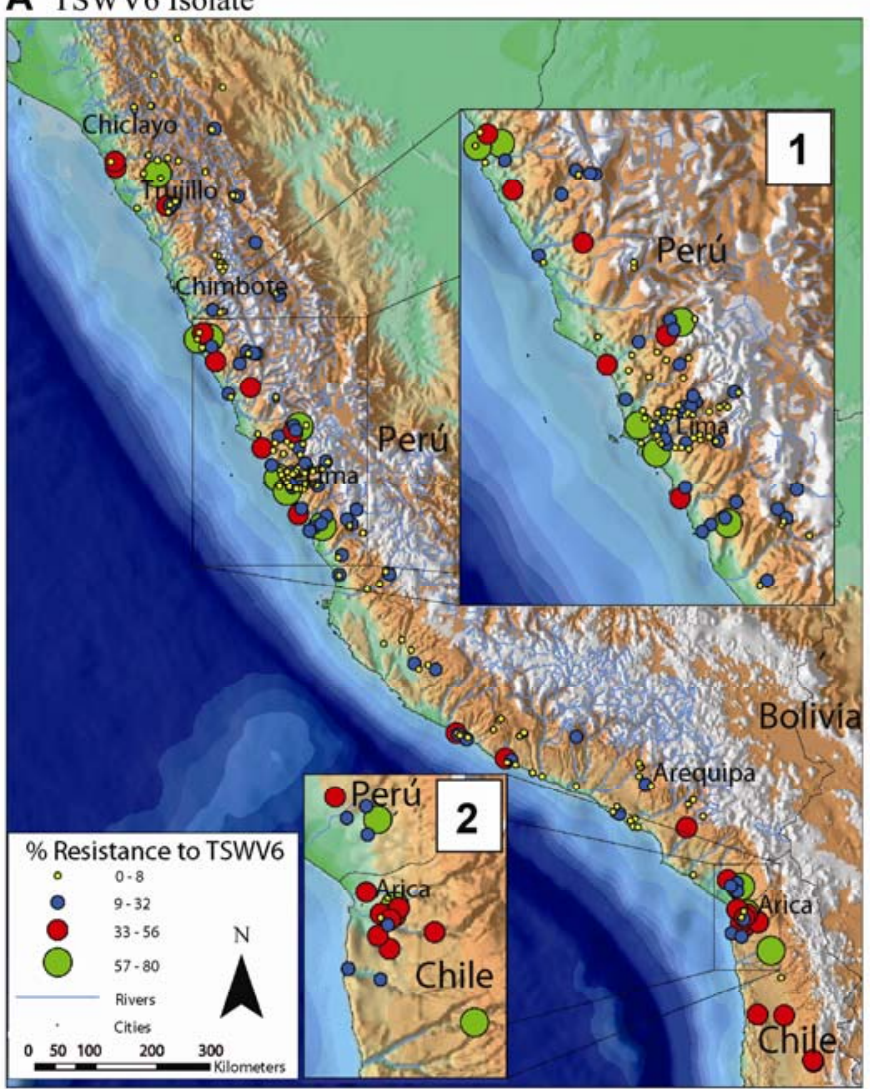

B $\mathrm{An}_{\mathrm{wa}}-1$ Isolate

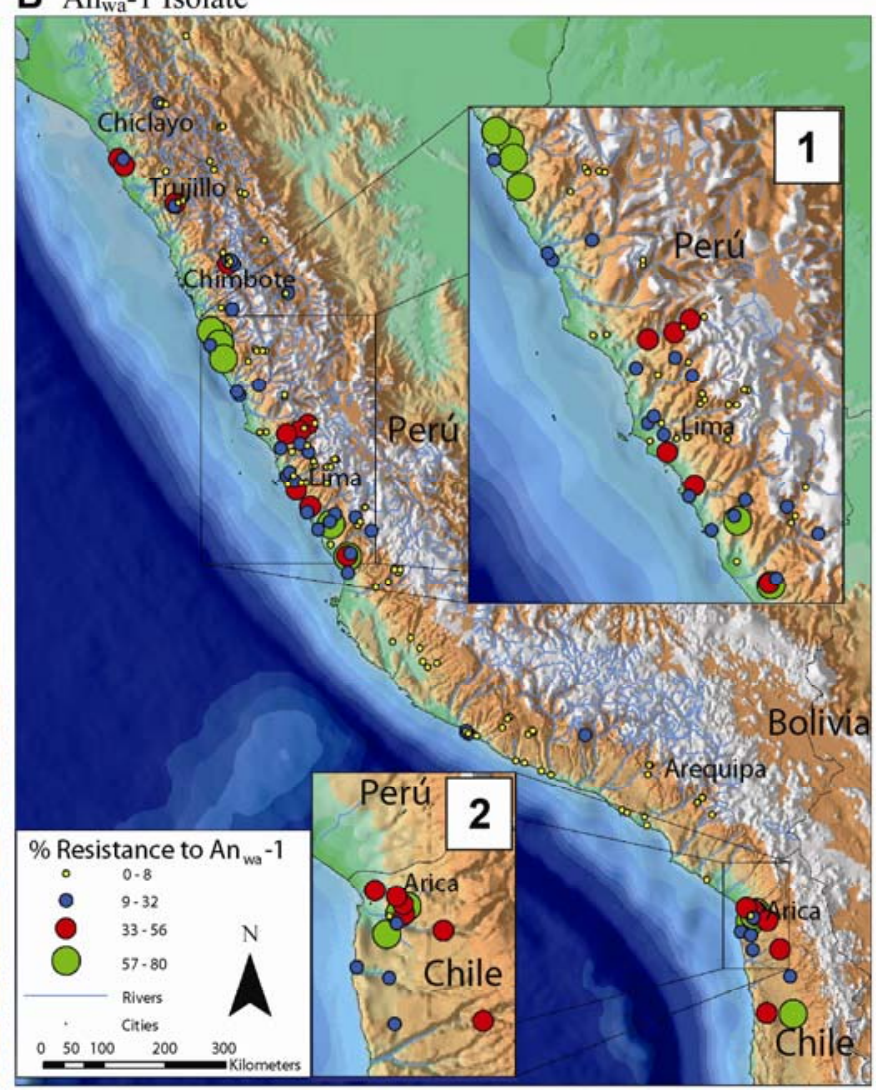

Fig. 2. Maps of Peru and Chile created with the Arc/GIS program showing the spatial distribution of the accessions collected according to the global positioning coordinates. There is sufficient passport data to map 256 accessions tested with the A, Tomato spotted wilt virus (TSWV) Hawaiian isolate (TSWV 6 ) and B, 158 tested with anemone isolate $\left(\mathrm{An}_{\mathrm{wa}}-1\right)$ for a total of 260 between the two isolates. These accessions are maintained at the United States Department of Agriculture Research Service (USDA) located at the Cornell University Geneva, NY campus, Tomato Genetics Resource Center (TGRC), University of California, Davis, and TGRC Core accessions. Accessions are presented with their percent resistance (dot size and color coded). Specific accessions indicated on this map are those with city or region as well as country included in its source information in Table 2.

with DI RNAs which still produced disease after almost 20 years of mechanically transferring the isolates. Norris (31) first identified resistance in L. peruvianum, and Finlay $(11,12)$ differentiated $S w_{1}{ }^{\mathrm{a}}, S w_{1}{ }^{\mathrm{b}}$, $s w_{2}, s w_{3}$, and $s w_{4}$ utilizing mechanical inoculations. Additionally, $S w-5$ was named $(54,57)$, mapped $(55,56)$, and subsequently cloned (13) based on TSWV mechanical inoculation methods. We have been working with a new source of TSWV resistance derived from $L$. chilense $(6,48)$ using mechanical inoculation. This resistance source has demonstrated its utility under naturally high TSWV pressure in repeated field trials (6,48; unpublished data). These studies demonstrate that mechanical serial inoculation methods have provided reliable data. In the study reported here, we were unable to identify discernable phenotypic differences in the TSWV symptoms of the controls over the duration of the screening with either TSWV6 or $\mathrm{An}_{\mathrm{wa}}-1$, which is in accordance with our previous studies $(6,54-58)$.

TSWV evaluations using both ELISA and visual techniques. Both isolates (TSWV6 and $\mathrm{An}_{\mathrm{wa}}-1$ ) produced a range of systemic symptoms throughout the study and within a given inoculation, such as tip blight, necrotic and chlorotic lesions, epinasty, and stunting in both the controls and L. peruvianum plants. However, both TSWV6 and $\mathrm{An}_{\mathrm{wa}}-1$ required more time before the typical symptoms appeared in either the controls or the tested accessions than in our past experiments with either 85-9 or HR-1 (6,54,56-58; unpublished data). Using these isolates, we found that many of the inoculated plants indicated TSWV infection according to ELISA and did not always indicate visual symptoms 
for several weeks post inoculation. Utilizing ELISA as a selection technique has been used for a number of TSWV resistance experiments $(6,34,54-58)$. The conservative ELISA threshold suggested by Sutula et al. (59) has demonstrated a reasonable method of selecting infected plant material $(6,34,54-58)$.

Differences in controls between the TSWV6 and $A n_{w a}-1$ TSWV isolates. The reaction of $89 \mathrm{R}$ and $89 \mathrm{~S}$ controls demonstrated that TSWV6 was significantly $(P$ value $<0.0001)$ more virulent than $\mathrm{An}_{\mathrm{wa}}-1$. It was unexpected to have $73 \%$ of the $89 \mathrm{R}$ plants infected using $\mathrm{An}_{\mathrm{wa}}-1$ because $\mathrm{Sw}-5$ genotypes reportedly were resistant to $\mathrm{An}_{\mathrm{wa}}-1$ (25). Using typical TSWV isolates from various areas within the United States, we have found that the degree of resistance provided by $S w-5(S w-5 / S w-5$ or $\left.S w-5 / S w-5^{+}\right)$results in 80 to $100 \%$ of plants being free of TSWV infection (6,57,58; unpublished data). This study shows that $\mathrm{An}_{\mathrm{wa}}-1$ overcomes $89 \mathrm{R}$ significantly ( $P$ value $<0.0001)$ less often than it infects $89 \mathrm{~S}$ plants but at a much greater frequency than isolates we have used for past studies $(6,57)$, suggesting that the TSWV isolates used in the Latham and Jones study (25) had a propensity to overcome $S w-5$ in their study.

Accession resistance responses to TSWV6 and $\mathbf{A n}_{\mathrm{wa}} \mathbf{- 1}$. Several studies $(20,22,31)$ have found, when testing two unique isolates, that a given accession may demonstrate resistance to one and not another. Fifty-four accessions tested with both isolates showed resistance to one isolate (Table 2). It is reasonable to attribute some of the purported resistance response in the $L$. peruvianum accessions when working with $\mathrm{An}_{\text {wa }}-1$ to individual plants escaping infection because $5 \%$ of the $89 \mathrm{~S}$ controls were not infected by this isolate (Table 1). However, 11 of the 23 accessions with a resistance response to $\mathrm{An}_{\mathrm{wa}}-1$ had more than $10 \%$ of the plants not infected. Conversely, more than $10 \%$ of the plants (13 of the 31 accessions), showed resistance to TSWV6 but not to $\mathrm{An}_{\mathrm{wa}}-1$ (Table 2).

The unilateral resistance response (either TSWV6 or $\mathrm{An}_{\mathrm{wa}}-1$ ) of 54 accessions suggests multiple alleles or genes for resistance to TSWV within the $L$. peruvianum germplasm collections. This theory is further supported by studies of smaller populations showing unique $L$. peruvianum resistance responses to varying TSWV isolates $(20,22,31)$.

Geographical distribution of resistant to TSWV. The unusually high percentage $(\geq 81 \%)$ of the accessions with $>8 \%$ plants free of infection to either isolate found in northern Chile and southern Peru is intriguing (Table 2; Fig. 2, inserts 2 and south to map edge, about $325 \mathrm{~km}$ ). When examining the passport data of the accessions from this area, we found that they were not all collected in the same year or by the same collection parties. Without additional in-depth studies, it is not possible to determine why apparently higher levels of TSWV resistance have been identified in this area. Curiously, our data suggest that, immediately north of this region, a general paucity of TSWV resistance occurs. However, in the northern part of Peru (near Lima and to the north), there are "pockets" of multiple accessions with higher levels of resistance to both isolates. For instance, the Culebras, Peru region (Fig. 2, northwest corner of inset 1) has a set of four accessions (PI 126944, PI 126945, PI 126946, and PI 127839) with relatively higher levels of resistance to both isolates used in this study. Additionally, the Ecuadorian accessions from three locations in two adjacent provinces (not found in Fig. 2), PI 128663, PI 129152, PI 129147, and PI 143679, tested only with TSWV6, indicated high percentages of virus-free plants ranging from 33 to $54 \%$.

TSWV resistance distribution within the germplasm collections. The USDA and TGRC are collaborative centers and each collection is somewhat representative of the wider indigenous geographical range of the species (Table 2). Hence, it is natural to expect that TSWV resistance would be somewhat equally distributed between these two germplasm collections. The TGRC Core collection was carefully selected using multiple criteria to represent as much $L$. peruvianum diversity as possible. Therefore, it would be expected to have approximately the same percentages of TSWV resistance as found in the greater collections. However, we found the USDA and "noncore" TGRC collections had a similar percentage of accessions with plants that had a resistant response to either TSWV6 or $\mathrm{An}_{\mathrm{wa}}-1$ (Fig. 1B), whereas the TGRC Core has significantly less $(P<$ 0.0001). Furthermore, the USDA collection had significantly more $(P<0.0001)$ resistant plants per individual accession (Fig. 1A).

Previous studies of TSWV resistance within $L$. peruvianum accessions. Of the 285 accessions used in our study, 23 (Table 2) had been screened for TSWV resistance in other studies $(4,7,15,20,22-24,34,47,49$, $50,52-54,57,58)$. Of those 23 accessions, 13 were tested with both isolates, 9 with TSWV6, and 1 with $\mathrm{An}_{\mathrm{wa}}-1$ (Table 2).

Of the 13 accessions previously reported $(4,7,20,22,34,47,49,50,52,58)$ and tested with both isolates, 12 (PI 126929, PI 126930, PI 126441, PI 126444, PI 126928, PI 126935, PI 126944, PI 126945, PI 126946, PI 128652, LA 111, and LA 444) had no less than $15 \%$ of the plants, within an accession, resistant to at least one of the two isolates (Table 2). Of the 13 accessions tested with both isolates, 3 indicated dramatic differences in the resistance responses between the isolates. Two of these three accessions (LA 111 and PI 126929) had little to no resistance to TSWV6 (1 and $0 \%$, respectively) and one (PI 126444) indicated $23 \%$ of the plants resistant to TSWV6 and 9\% resistant to $\mathrm{An}_{\mathrm{wa}} \mathrm{a}^{-1}$ (Table 2). PI 126444 and LA 111 had been tested previously with multiple isolates. Stevens et al. (58) tested PI 126444 with three isolates and found a similar level of resistance response between the isolates. In contrast to our results, Iizuka et al. (20) found that $100 \%$ of the LA 111 plants were resistant to two isolates of TSWV whereas $16 \%$ of the plants were susceptible to two other isolates.

Of the 23 accessions examined in previous TSWV resistance studies $(7,15,20,24$, 27,34,47,49,50,52,57), 10 were tested with either TSWV6 or $\mathrm{An}_{\mathrm{wa}}-1$ (Table 2). Only three plants of accession LA 441 (tested with TSWV6) germinated; however, the remaining nine accessions (PI 128654, PI 128657, PI 128659, PI 128660, PI 129146, PI 143679, PI 251311, LA 372, and LA 385 ) had sufficient plants per accession to provide clear evidence of possible resistance (Table 2). Finding TSWV resistance in these accessions is in agreement with the previous reports $(7,15,24,27,34,47,49$, $50,52,57)$. However, little or no resistance was found in LA 372 and LA 385 to TSWV6 and $\mathrm{An}_{\mathrm{wa}}-1$, respectively. Both of these accessions demonstrated resistance to three of four isolates used by Iizuka et al. (20).

Tomato lines purported to derive their resistance from $L$. peruvianum. Four tomato cultivars or lines (Anahu, UPV 1, UPV 32, and Stevens) attribute their purported TSWV resistance to $L$. peruvianum. $\mathrm{Cv}$. Anahu (15) was reported to have the TSWV resistance gene $S w-1$ from PI 128657 (14). Previous research has found Anau to be easily infected with various TSWV isolates; this study had $40 \%$ of the plants free of infection using TSWV6 on PI $128657(24,33,34)$. Finding that $40 \%$ of the plants resisted TSWV6 suggests the existence of alleles differing from $S w-1$, or it is quantitatively inherited. Our results, in combination with the past studies of $S w-1$, do suggest that a given accession may harbor multiple genes for resistance to TSWV.

The tomato lines UPV 1 and UPV 32 were derived from $L$. peruvianum accession PE-18 (46). UPV 1 is purported to have a single dominant unnamed TSWV resistance gene suggesting allelism to $S w-5$ (46). UPV 32 was identified as having a single resistance gene not allelic to $S w-5$ named $S w-6$ (45). We did not have PE-18 to screen; however, this $L$. peruvianum accession was reported to be from Huallanca, Ancash, Peru, where three accessions used in our study originated (LA 1982, LA 2561, and LA 2562; Table 2). Two of these accessions (LA 2561 and LA 2562) were tested with our isolates, resulting in three to four times the number of plants with resistance to $\mathrm{An}_{\mathrm{wa}}-1$ than to TSWV6. LA 1982 was tested only with 
TSWV6 and it had little resistance (2\%), similar to the 3 and $5 \%$ found in LA 2561 and LA 2562, respectively. Thus, all three of the accessions from Huallanca, Ancash, Peru indicate little resistance to TSWV6, the isolate which completely overcomes $S w-5$. These data suggest agreement with the results of PE-18 (45-47) with an $S w-5$ like response. We have tested UPV 32 with TSWV5 from Hawaii and found it fully susceptible (unpublished data). UPV 1 has not been tested in our program.

The last reported cultivar with purported L. peruvianum-derived TSWV resistance is Stevens $(53,57)$. This cultivar is one of two separately derived sources of $S w-5$ (5). There is evidence that $S w-5$ (from Stevens) originated from one of the L. peruvianum accessions PI 126928, PI 126929, PI 126944, PI 128645, PI 128654, or PI 129109 (57). We were able to include all except PI 129109 in our study. PI 126928, PI 126929, PI 126944, and PI 128645 were tested with both isolates while PI 128654 was tested only with TSWV6. Interestingly, all of these accessions were among the most resistant accessions tested (Table 2). PI 128654 is considered a strong candidate as the source of $S w-5$ (57). However, our data provide an alternative candidate accession, PI 126929, which did not indicate resistance to TSWV6, although it had $44 \%$ of the plants indicate $\mathrm{An}_{\mathrm{wa}}-1$ resistance. Even with two lines of evidence for the possible origin of $S w-5$, it is possible that all six accessions have $S w-5$. Interestingly, at least five of the six accessions have differing resistance relative to $S w-5$, in that a substantial number of plants were resistant to TSWV6 in those five accessions.

The value of the $\boldsymbol{S w - 5}$ gene. There is clear evidence that variant isolates of TSWV can overcome $S w-5 \quad(1,7,47,61)$. Furthermore, studies have demonstrated that tospoviruses can readily overcome both pathogen-derived resistance and natural resistance through reassortment of the TSWV genome $(17,36,37)$. With these two lines of evidence in mind, the question arises as to why $S w-5$ has still been used worldwide, providing a high level of resistance in most areas where utilized. In regions where a TSWV isolate has overcome $S w-5$, two related conditions have been noted. First, TSWV6 came from a situation where virtually $100 \%$ of the $S w-5^{+} / S w-5^{+}$ genotypes were naturally infected; thus, the disease pressure was extremely high. Second, where clear case histories can be identified; there often have been yeararound cropping of $S w-5$ genotype tomato with consistently "high" Tospovirus pressure. Curiously, TSWV6-like isolates have not been found in Hawaii since $S w-5$ was no longer used in the area. In fact, tomato plants with $S w-5$ were planted again several years later in the same region and $S w$ 5 provided its normal level of resistance (J. Cho, personal communication). This " $\mathrm{Sw}-5$ rest" allowed the indigenous tospovirus to revert to an "environmentally fit" tospovirus that did not include the ability to readily overcome $S w-5$.

These anecdotal observations, coupled with the data we obtained when using $\mathrm{An}_{\mathrm{wa}}-1$, opens the question to the environmental "fitness" of TSWV isolates that overcome $S w-5 . \mathrm{An}_{\mathrm{wa}}-1$ was used in the experiments, demonstrating that TSWV isolates could be selected to overcome TSWV resistance (25). If isolates overcoming $S w-5$ are generally less "environmentally fit," it would explain why growers who seasonally utilize $S w-5$ genotype cultivars where TSWV pressure is high expect as much as $20 \%$ of their crop to be infected with the virus. However, regions that have consistently high TSWV pressure and never allow a rest from $S w-5$ are purported to be areas having troubles with TSWV overcoming this resistance. In considering the evidence that tospoviruses can quickly and easily "reshuffle" their genome, developing stable TSWV resistance may be a challenge $(17,36,37)$. However, the slow adaptation of the virus to overcome $S w-5$ coupled with information that this event is documented mostly in areas where TSWV is infecting nearly $100 \%$ of known susceptible lines are in complete harmony with Leach et al. (26). Their results suggest that environmental fitness of this pathogen is directly related to the durability of resistance genes. The study we are reporting here was conducted to search for additional genes equal to or of even greater value than $S w-5$ in the L. peruvianum germplasm.

Our results demonstrate that there is additional tospovirus resistance to be found in the L. peruvianum germplasm. It is unclear as to how much of this resistance is quantitative or qualitative in nature. However, there is evidence that both inheritance patterns for TSWV resistance can be found within $L$. peruvianum germplasm. $S w-5$ was identified from this species (57) and, in an unpublished study, we recently have endeavored to introgress resistance to TSWV6 from PI 128660 into tomato. We found that the high level of tospovirus resistance seems to be quantitative in nature. It becomes clear that mining additional TSWV resistance from $L$. peruvianum is realistic but should be approached with full awareness that both successful introgression of resistance and failure of that objective is a possible outcome.

Making interspecific crosses with $L$. peruvianum is challenging $(16,35)$. Therefore, an optimal strategy that may produce functional, transferable, TSWV resistance would be to focus on accessions that appear to provide resistance to one isolate and not the other. For example, LA 2808 indicated resistance to $\mathrm{An}_{\mathrm{wa}}-1$ and $\mathrm{G} 30027$ to TSWV6, but neither accession suggested resistant to the opposite isolates (Table 2). Even with the obvious hazard of incorporating isolate-specific resistance, it may be that this resistance is more heritable and different from each other. Attempts have been made to introgress the resistance of PI 128660 and other previously reported accessions into tomato by several breeding programs, resulting in no known long-term usable resistance incorporated into elite cultivars. Identifying more single genes such as $S w-5$ for breeding potentially can provide a suite of resistant genes. Thus, searching for accessions demonstrating clear segregating type resistance may produce genes more readily introgressed with more utility than the resistance introgressions previously attempted.

Although there is clear evidence that tospoviruses can readily overcome various types of resistance $(1,17,25,36,37)$, the environmental fitness of such a tospovirus may prove difficult for an epidemic type of behavior of a new isolate (26). Nevertheless, carefully planned deployment of tospovirus resistance, either natural or pathogen derived, should be part of the overall management of a crop in conditions where this virus is prevalent.

\section{ACKNOWLEDGMENTS}

This study was partially supported by funding from the USDA Tomato Germplasm Evaluation Committee. The seed from the germplasm collection used in this study was provided by TGRC at University of California, Davis and the USDA Agricultural Research Service plant genetic resources unit, Cornell University, Geneva, NY. We thank R. D. Horrocks, Plant and Animal Sciences, Brigham Young University, Provo, Utah, for help with the statistics of this article.

\section{LITERATURE CITED}

1. Aramburu, J., and Martí, M. 2003. The occurrence in north-east Spain of a variant of Tomato spotted wilt virus (TSWV) that breaks resistance in tomato (Lycopersicon esculentum) containing the $S w-5$ gene. Plant Pathol. 52:407.

2. Aramburu, J., and Rodriguez, M. 1999 Evaluation of commercial Lycopersicon esculentum hybrids for resistance to tomato spotted wilt tospovirus (TSWV) in Spain. J. Hortic. Sci. Biotechnol. 74:743-747.

3. Aramburu, J., Rodriguez, M., and Ariño, J. 2000. Effect of Tomato spotted wilt tospovirus (TSWV) infection on the fruits of tomato ( $L y$ copersicon esculentum) plants of cultivars carrying the $S w-5$ gene. J. Phytopathol. 148:569-574.

4. Boiteux, L. S., and Giordano, L. de B. 1992 Screening Lycopersicon germplasm for resistance to a Brazilian isolate of spotted wilt virus (TSWV). Tomato Genet. Coop. Rep. 42:13-14.

5. Brommonschenkel, S. H. 1996. Genetic mapping and progress towards the positional cloning of the $S w-5$ Tomato spotted wilt virus (TSWV) resistance gene in tomato (Lycopersicon esculentum Mill.). Ph.D. dissertation, Cornell University, Ithaca, NY.

6. Canady, M. A., Stevens, M. R., Barineau, M S., and Scott, J. W. 2001. Tomato spotted wilt virus (TSWV) resistance in tomato derived from Lycopersicon chilense Dun. LA 1938. Euphytica 117:19-25.

7. Cho, J. J., Custer, D. M., Brommonschenkel, S. H., and Tanksley, S. D. 1996. Conventional breeding: Host-plant resistance and the use of molecular markers to develop resistance to Tomato spot wilt virus in vegetables. Acta Hortic. 431:367-378.

8. Cho, J. J., Mau, R. F. L., German, T. L., Hart- 
mann, R. W., Yudin, L. S., Gonsalves, D., and Provvidenti, R. 1989. A multidisciplinary approach to management of Tomato spotted wilt virus in Hawaii. Plant Dis. 73:375-383.

9. Cho, J. J., Mau, R. F. L., Gonsalves, D., and Mitchell, W. C. 1986. Reservoir weed hosts of Tomato spotted wilt virus. Plant Dis. 70:10141017.

10. Cupertino, F. P., Ávila, A. C., Araújo, M. T., and Maluf, W. R. 1986. Fontes de resistência ao virus de vira-cabeça em Lycopersicon. Fitopatol. Bras. 11:330-331.

11. Finlay, K. W. 1952. Inheritance of spotted wilt virus resistance in the tomato. I. Identification of strains of the virus by the resistance or susceptibility of tomato species. Aust. J. Sci. Res. Ser. B Biol. Sci. 5:303-314

12. Finlay, K. W. 1953. Inheritance of spotted wilt resistance in the tomato. II. Five genes controlling spotted wilt resistance in four tomato types. Aust. J. Biol. Sci. 6:153-163.

13. Folkertsma, R. T., Spassova, M. I., Prins, M., Stevens, M. R., Hille, J., and Goldbach, R. W. 1999. Construction of a bacterial artificial chromosome (BAC) library of Lycopersicon esculentum cv. Stevens and its application to physically map the $S w-5$ locus. Mol. Breed. 5:197-207.

14. Gilbert, J. C., and McGuire, D. C. 1952. Root knot resistance in commercial type tomatoes in Hawaii. Proc. Am. Soc. Hortic. Sci. 60:401-411.

15. Gilbert, J. C., and Tanaka, J. S. 1971. 'Anahu,' an outstanding hybrid maker. Hawaii Farm Sci. 20:6-7.

16. Gordillo, L. F., Jolley, V. D., Horrocks, R. D., and Stevens, M. R. 2003. Interactions of BA, $\mathrm{GA}_{3}$, NAA, and surfactant on interspecific hybridization of Lycopersicon esculentum $\times L$. chilense. Euphytica 131:15-23.

17. Hoffmann, K., Qiu, W. P., and Moyer, J. W. 2001. Overcoming host- and pathogenmediated resistance in tomato and tobacco maps to the M RNA of Tomato spotted wilt virus. Mol. Plant-Microbe Interact. 14:242-249.

18. Hutton, E. M., and Peak, A. R. 1949. Spotted wilt resistance in the tomato. J. Inst. Agric. Sci. 15:32-36.

19. Ie, T. S. 1982. A sap-transmissible, defective form of Tomato spotted wilt virus. J. Gen. Virol. 59:387-391.

20. Iizuka, N., Boiteux, L. S., Giordano, L. de B., Nagata, T., and Dusi, A. N. 1993. Sources of resistance to Tomato spotted wilt virus (TSWV) in wild Lycopersicon species. Tomato Genet. Coop. Rep. 43:20-22.

21. Inoue-Nagata, A. K., Kormelink, R., Nagata, T., Kitajima, E. W., Goldbach, R., and Peters, D. 1997. Effects of temperature and host on the generation of Tomato spotted wilt virus defective interfering RNAs. Phytopathology 87:1168-1173.

22. Jordá, C., Abad, P., Juarez, M., MartinezHerrera, D., Sanchez, F., Ponz, F., Díez, M. J., and Nuez, F. 1993. Differential behavior of TSWV isolates on tomato and related species. Tomato Genet. Coop. Rep. 43:24-26.

23. Kumar, N., and Irulappan, I. 1992. Inheritance of resistance to spotted wilt virus in tomato (Lycopersicon esculentum Mill.). J. Genet. Breed. 46:113-117.

24. Kumar, N. K. K., Ullman, D. E., and Cho, J. J. 1993. Evaluation of Lycopersicon germ plasm for Tomato spotted wilt tospovirus resistance by mechanical and thrips transmission. Plant Dis. 77:938-941.

25. Latham, L. J., and Jones, R. A. C. 1998. Selection of resistance breaking strains of Tomato spotted wilt tospovirus. Ann. Appl. Biol. 133:385-402.

26. Leach, J. E., Cruz, C. M. V., Bai, J., and Leung, H. 2001. Pathogen fitness penalty as a predictor of durability of disease resistance genes. Annu. Rev. Phytopathol. 39:187-224.

27. Maluf, W. R., Toma-Braghini, M., and Corte,
R. D. 1991. Progress in breeding tomatoes for resistance to tomato spotted wilt. Braz. J. Genet. 14:509-525.

28. McMichael, L. A., Persley, D. M., and Thomas, J. E. 2000. The first record of a serotype IV Tospovirus in Australia. Australas. Plant Pathol. 29:149.

29. Momol, M. T., Olson, S. M., Funderburk, J. E., Stavisky, J., and Marois, J. J. 2004. Integrated management of tomato spotted wilt on fieldgrown tomatoes. Plant Dis. 88:882-890.

30. Moriones, E., Aramburu, J., Riudavets, J., Arno, J., and Lavina, A. 1998. Effect of plant age at time of infection by Tomato spotted wilt tospovirus on the yield of field-grown tomato. Eur. J. Plant Pathol. 104:295-300

31. Norris, D. O. 1946. The strain complex and symptom variability of Tomato spotted wilt virus. Aust. Counc. Sci. Ind. Res. Bull. 202:1-62.

32. Parrella, G., Gognalons, P., Gebre-Selassiè, K., Vovlas, C., and Marchoux, G. 2003. An update of the host range of Tomato spotted wilt virus. J. Plant Pathol. 85:227-264.

33. Paterson, R. G. 1987. Epidemiology and genetic resistance in tomato to the Tomato spotted wilt virus in Arkansas. Thesis, University of Arkansas, Fayetteville.

34. Paterson, R. G., Scott, S. J., and Gergerich, R. C. 1989. Resistance in two Lycopersicon species to an Arkansas isolate of Tomato spotted wilt virus. Euphytica 43:173-178.

35. Picó, B., Herraiz, J., Riuz, J. J., and Nuez, F. 2002. Widening the genetic basis of virus resistance in tomato. Sci. Hortic. 94:73-89.

36. Qiu, W. P., Geske, S. M., Hickey, C. M., and Moyer, J. W. 1998. Tomato spotted wilt tospovirus genome reassortment and genome segmentspecific adaptation. Virology 244:186-194.

37. Qiu, W. P., and Moyer, J. W. 1999. Tomato spotted wilt tospovirus adapts to the TSWV N gene-derived resistance by genome reassortment. Phytopathology 89:575-582.

38. Resende, L. V., Maluf, W. R., Figueira, A. de R., and Resende, J. T. V. 2000. Correlations between symptoms and DAS-ELISA values in two sources of resistance against Tomato spotted wilt virus. Braz. J. Microbiol. 31:135-139.

39. Resende, R. de O., de Haan, P., de Ávila, A. C., Kitajima, E. W., Kormelink, R., Goldbach, R., and Peters, D. 1991. Generation of envelope and defective interfering RNA mutants of Tomato spotted wilt virus by mechanical passage. J. Gen. Virol. 72:2375-2383.

40. Resende, R. de O., de Haan, P., Vandevossen, E., de Ávila, A. C., Goldbach, R., and Peters, D. 1992. Defective interfering L-RNA segments of Tomato spotted wilt virus retain both virus genome termini and have extensive internal deletions. J. Gen. Virol. 73:2509-2516.

41. Rick, C. M., and Hunt, D. 1961. Improved seed germination with the use of sodium hypochlorite. Tomato Genet. Coop. Rep. 11:22.

42. Riley, D. G., and Pappu, H. R. 2000. Evaluation of tactics for management of thripsvectored Tomato spotted wilt virus in tomato. Plant Dis. 84:847-852.

43. Riley, D. G., and Pappu, H. R. 2004. Tactics for management of thrips (Thysanoptera: Thripidae) and Tomato spotted wilt virus in tomato. J. Econ. Entomol. 97:1648-1658.

44. Roselló, S., Díez, M. J., Lacasa, A., Jordá, C., and Nuez, F. 1997. Testing resistance to TSWV introgressed from Lycopersicon peruvianum by artificial transmission techniques. Euphytica 98:93-98.

45. Roselló, S., Díez, M. J., and Nuez, F. 1998. Genetics of Tomato spotted wilt virus resistance coming from Lycopersicon peruvianum. Eur. J. Plant Pathol. 104:499-509.

46. Roselló, S., Ricarte, B., Díez, M. J., and Nuez, F. 2001. Resistance to Tomato spotted wilt virus introgressed from Lycopersicon peruvianum in line UPV 1 may be allelic to $S w-5$ and can be used to enhance the resistance of hy- brids cultivars. Euphytica 119:357-367.

47. Roselló, S., Soler, S., Díez, M. J., Rambla, J. L., Richarte, C., and Nuez, F. 1999. New sources for high resistance of tomato to the Tomato spotted wilt virus from Lycopersicon peruvianum. Plant Breed. 118:425-429.

48. Scott, J. W., Stevens, M. R., and Olson, S. M 2005. An alternative source of resistance to Tomato spotted wilt virus. Tomato Genet. Coop. Rep. 55:40-41

49. Segeren, M. I., Siqueira, W. J., Sondahl, M. R., Lourenção, A. L., Filho, H. P. M., and Nagai, H. 1993. Tomato breeding: 2. Characterization of $F_{1}$ and $F_{2}$ hybrid progenies of Lycopersicon esculentum $\times L$. peruvianum and screening for virus and insect resistance. Rev. Bras. Genet. 16:773-783.

50. Segeren, M. I., Sondahl, M. R., Siqueira, W. J., Filho, H. P. M., Nagai, H., and Lourenção, A L. 1993. Tomato breeding: 1. Embryo rescue of interspecific hybrids between Lycopersicon esculentum Mill. and L. peruvianum (L.) Mill Rev. Bras. Genet. 16:367-380.

51. Sialer, M. M. F., Lanave, C., Padula, M., Vovlas, C., and Gallitelli, D. 2002. Occurrence of two distinct Tomato spotted wilt virus subgroups in southern Italy. J. Plant Pathol. 84:145-152.

52. Smith, P. G. 1944. Reaction of Lycopersicon spp. to spotted wilt. Phytopathology 34:504 505.

53. Stevens, J. M. 1964. Tomato breeding. Project Report W-Vv1. Agricultural Technical Services, Republic of South Africa.

54. Stevens, M. R. 1993. Studies on inheritance of resistance to Tomato spotted wilt virus in the genus Lycopersicon. Ph.D. dissertation, University of Arkansas, Fayetteville.

55. Stevens, M. R., Heiny, D. K., Rhoads, D. D. Griffiths, P. D., and Scott, J. W. 1996. A linkage map of the Tomato spotted wilt virus resistance gene $S w-5$ using isogenic lines and an interspecific cross. Acta Hortic. 431:385-392.

56. Stevens, M. R., Lamb, E. M., and Rhoads, D. D. 1995. Mapping the $S w-5$ locus for Tomato spotted wilt virus resistance in tomatoes using RAPD and RFLP analyses. Theor. Appl. Genet. 90:451-456.

57. Stevens, M. R., Scott, S. J., and Gergerich, R. C. 1992. Inheritance of a gene for resistance to Tomato spotted wilt virus (TSWV) from Lycopersicon peruvianum Mill. Euphytica 59:9-17.

58. Stevens, M. R., Scott, S. J., and Gergerich, R. C. 1994. Evaluation of seven Lycopersicon species for resistance to Tomato spotted wilt virus (TSWV). Euphytica 80:79-84.

59. Sutula, C. L., Gillett, J. M., Morrissey, S. M. and Ramsdell, D. C. 1986. Interpreting ELISA data and establishing the positive-negative threshold. Plant Dis. 70:722-726.

60. Thomas-Carroll, M. L., and Jones, R. A. C. 2003. Selection, biological properties and fitness of resistance-breaking strains of Tomato spotted wilt virus in pepper. Ann. Appl. Biol. 142:235-243.

61. Thompson, G. J., and van Zijl, J. J. B. 1996. Control of Tomato spotted wilt virus in tomatoes in South Africa. Acta Hortic. 431:379-384.

62. van Zijl, J. J. B., Bosch, S. E., and Coetzee, C. P. J. 1986. Breeding tomatoes for processing in South Africa. Acta Hortic. 194:69-75.

63. Watterson, J. C. 1993. Development and breeding of resistance to pepper and tomato viruses. Pages 80-101 in: Resistance to Viral Diseases of Vegetables. M. M. Kyle, ed. Timber Press, Portland, OR.

64. Wenholz, H. 1939. Spotted wilt of tomatoes Breeding for resistance. Hawkesbury Agric. Coll. J. 36:103.

65. Williams, L. V., Lambertini, P. M. L., Shohara, K., and Biberbost, E. B. 2001. Occurrence and geographical distribution of Tospovirus species infecting tomato crops in Argentina. Plant Dis. 85:1227-1229. 